\title{
The effectiveness and safety of cupping therapy for stroke survivors: A systematic review and meta-analysis of randomized controlled trials
}

\author{
Mikyung Kim ${ }^{1}$, Chang-ho $\mathrm{Han}^{2 *}$ \\ 'Department of Internal Medicine, College of Korean Medicine, Sangji University \\ ${ }^{2}$ Department of Internal Medicine, College of Korean Medicine, Dongguk University
}

\begin{abstract}
Objectives: ncluding stroke. The aim of this study was to systematically review the clinical evidence of CT for stroke.

Methods: To identify randomized controlled trials (RCTs) reporting the effectiveness and/or safety of CT, seven databases including PubMed, EMBASE, and Cochrane Library were searched for articles published from January 2000 to February 2021 without language restrictions. Meta-analysis was performed using Review Manager 5.4 software and the results were presented as mean difference (MD) or standard mean difference (SMD) for continuous variables and odds ratio (OR) for diverse variables with 95\% confidence intervals (CIs). Assessment of the methodological quality of the eligible trials was conducted using the Cochrane Collaboration tool for risk of bias in RCTs.

Results: Twenty-two RCTs with 1653 participants were included in the final analysis. CT provided additional benefit in improving upper limb motor function (Fugl-Meyer assessment for upper limb motor function, MD 6.91, 95\% CI 4.64 to $1.67, \mathrm{P}<0.00001$ ) and spasticity (response rate, $\mathrm{OR} 3.28,95 \% \mathrm{CI} 1.31$ to $8.22, \mathrm{P}=0.08$ ) in stroke survivors receiving conventional medical treatment. These findings were supported with a moderate level of evidence. CT did not significantly increase the occurrence of adverse events.

Conclusions: This study demonstrated the potential of $\mathrm{CT}$ to be beneficial in managing a variety of complications in stroke survivors. However, to compensate for the shortcomings of the existing evidence, rigorously designed large-scale RCTs are warranted in the future.
\end{abstract}

$\overline{K e y}$ Words : Cerebrovascular diseases; Cupping; Meta-Analysis, Stroke; Systematic review

\section{Introduction}

Stroke is one of the leading causes of death worldwide, accounting for $10 \%$ of the world's mortality ${ }^{1)}$. Although stroke mortality is on the decline $^{1)}$, many stroke survivors still suffer from residual disability ${ }^{2}$. The aging of the population and the reduction in stroke deaths are expected to lead to further increases in the prevalence of stroke and socioeconomic burden in the future ${ }^{3)}$. Therefore, now is the time to pay attention not only to current stroke strategies mainly focused on emergent and intensive care in the acute stage but also to continuous monitoring and medical support for stroke survivors ${ }^{3)}$. Interest in complementary and alternative medicine, represented

\footnotetext{
- Received: 16 July 2021

- Revised : 3 August 2021

- Accepted : 5 November 2021

- Correspondence to: Chang-ho Han

Department of Internal Medicine, College of Korean Medicine, Dongguk University, Gyeongju, 38066, Republic of Korea Tel : +82-510-8449, Fax : +82-510-8450
} 
by traditional or folk medicine, has been increasing as one of the methods to satisfy the unmet needs of stroke survivors ${ }^{4}$.

Cupping therapy is a form of intervention that provides negative pressure on the skin by suctioning the air inside a cup and has been widely used in multiple cultures throughout the world $^{5,6)}$. In particular, it has one of the most commonly used interventions in modern East Asian traditional medicine to date ${ }^{5}$. Indications of cupping therapy covered almost all systems of the human body and mind in traditional medicine ${ }^{1)}$, including various complications experienced by stroke survivors ${ }^{6}$.

In 2010, Lee et $\mathrm{al}^{6)}$. published the results of a systematic review of the clinical literature on the effect of cupping therapy for stroke rehabilitation. However, at the time, only two uncontrolled observational studies and three randomized controlled trials (RCTs) comparing the effects of cupping therapy with different unproven techniques were identified in the search, so only a narrative review of the clinical evidence available at the time for this topic was possible.

Therefore, this study aimed to update the findings of the previous study ${ }^{6}$ by systematically reviewing currently available RCTs, performing a meta-analysis and providing evidence-based information for those considering the application of cupping therapy for stroke patients.

\section{Methods}

\section{Data sources and search strategy}

\section{1) Data sources}

Considering that the primary studies included in the previous systematic review on this topic $\left.{ }^{6}\right)$ have been published since 2000, we searched the following databases from 1 January 2000 to 16 February 2021: English (PubMed, EMBASE, and Cochrane Library), Chinese (China Academic Journals Full-text Database), and Korean databases (Science On, Korean Studies Information Service System, and Oriental Medicine Advanced Searching Integrated System). Twelve major Korean traditional medicine journals were also manually searched for relevant articles.

\section{2) Search strategy}

The search terms were used in the form of a combination of ("stroke" OR "cerebral infarction" OR "cerebral hemorrhage" OR "cerebrovascular disorder" OR "cerebrovascular accident" OR “apoplexy" OR "brain ischemia") AND (“cupping”), but modified to suit the condition of the search platform of each database in Korean, Chinese, and English (Supplement 1). There were no language restrictions. In addition to articles published in academic journals, thesis and conference abstracts were also included. Hand searches were also conducted for related references. This study was conducted according to the Preferred Reporting Items for Systematic reviews and Meta-Analyses 2020 (Supplement 2 \& 3).

\section{Study selection and eligibility criteria}

\section{1) Study selection}

$\mathrm{MK}$ and $\mathrm{CH}$ screened the retrieved records. The selection of potentially relevant articles for this study was decided after reading the abstract or full text of the articles. The two authors 
worked independently and any disagreement was resolved by discussion.

\section{2) Types of studies}

Parallel-design RCTs that investigated the effectiveness and safety of cupping therapy in stroke patients were selected. Observational studies, qualitative studies, animal studies, and literature reviews were not included. Clinical studies that were nonrandomized studies, crossover-design studies, before-and-after studies, or studies without a control group in comparison to the cupping therapy group were also excluded.

\section{3) Types of participants}

Studies that recruited patients who were confirmed to have stroke by clinical signs and symptoms supported by brain imaging were included. There were no other restrictions, such as age, sex, stroke type, or stroke stage. Studies including nonstroke patients were excluded. When more than one study used duplicated data were obviously from the same population, only the one study that provided more information was selected.

\section{4) Types of interventions}

We defined cupping therapy as an intervention that provides negative pressure on the skin by suctioning air inside a cup-shaped device (mainly made of plastic, glass, or bamboo). ${ }^{4)}$ Generally, cupping therapy is classified as wet cupping or dry cupping,5). Wet cupping refers to cupping with bloodletting puncture. In other words, it involves pricking the skin of the cupping site before suction and subsequently drawing blood in addition to cupping. Dry cupping involves only cupping without bleeding. This study included both types of cupping.

\section{5) Types of control interventions}

All types of control interventions other than cupping therapy were allowed as control interventions, including active control, sham or placebo control, and no cupping therapy. Therefore, both $\mathrm{A}$ versus $\mathrm{B}$ and $\mathrm{A}+\mathrm{B}$ versus $\mathrm{B}$ type studies were included.

\section{6) Types of outcome measures}

All types of clinical outcomes that evaluated the therapeutic effect and safety of cupping therapy for stroke patients were allowed. Reporting of the response rate based on predefined criteria was also allowed.

Outcomes showing the add-on effect of cupping therapy were also allowed. However, cases that provided only the combined effects of cupping plus other interventions in which the effects of cupping therapy could not be identified were excluded (e.g. cupping + acupuncture, cupping + herbal medicine, and cupping + bloodletting of noncupping sites).

When the outcomes were measured multiple times after the completion of planned treatment, the first measured value was preferentially adopted.

\section{Data extraction}

One of the authors (MK) extracted data, and another one $(\mathrm{CH})$ cross-checked the data. Disagreements were resolved by discussion between the two reviewers. The extracted data 
were as follows: number of participants in the final analysis, time-to-onset of stroke, stroke conditions, types and details of intervention, types of control intervention, outcome measures, and findings of each study.

\section{Data synthesis}

Meta-analysis was conducted when two or more studies provided quantifiable values. In the case of A versus B designs, data were combined when there were two or more studies using the same endpoints and control interventions. In the case of $\mathrm{A}+\mathrm{B}$ versus $\mathrm{B}$ designs, data were combined when there were two or more studies using the same endpoints. Cochrane Collaboration software (Review Manager (RevMan) Version 5.4 for Windows. The Cochrane Collaboration, 2020) was used for data synthesis and constructing forest plots. For continuous variables, the mean difference (MD) and its 95\% confidence interval (CI) were calculated using the inverse variance method. However, when the measurement tools were not identical across studies, the standard mean difference (SMD) was calculated. For binomial variables, odds ratios (ORs) and 95\% CIs were calculated using the Mantel-Haenszel method. Heterogeneity among trials was evaluated using Higgins' $\mathrm{I}^{2}$ test $^{7}$. The fixed effect model was preferentially adopted, but a random effect model was selected when the stroke conditions of the participants were not homogeneous or the statistical heterogeneity between studies was considerable $\left(\mathrm{I}^{2} \text { value } \geq 75 \%\right)^{8)}$. When heterogeneity was substantial, subgroup analysis was considered. Subgroup analysis was also performed based on the type of cupping therapy (dry or wet cupping).
When data from 10 or more studies were combined, the possibility of publication bias was assessed using a funnel plot.

\section{Risk of bias (RoB) assessment}

The Cochrane Collaboration tool for RoB in RCTs (RoB 2) was used to assess the methodological quality of the studies included in the meta-analysis. The RoB 2 consists of five domains: randomization process, deviations from intended interventions, missing outcome data, measurement of the outcome, and selection of the reported results ${ }^{9}$. Based on the evaluation results for each domain, the overall RoB for each study was judged to be one of the three levels including low, high, or some concerns. The two authors independently evaluated the RoB of individual studies, and any disagreements were resolved through discussion.

\section{Level of evidence}

The certainty in evidence from the meta-analysis was assessed by the methodology of the grading of recommendations, assessment, development, and evaluation (GRADE) ${ }^{10)}$. Meta-analysis data were imported into GRADEpro, web-based software, and the quality of evidence was rated based on the following criteria: study design, RoB, inconsistency, indirectness, imprecision, and other considerations including publication bias. Synthesizing the ratings of each criterion, the final level of evidence was decided to be high, moderate, low, or very low ${ }^{10)}$.

\section{Results}

\section{Study selection}




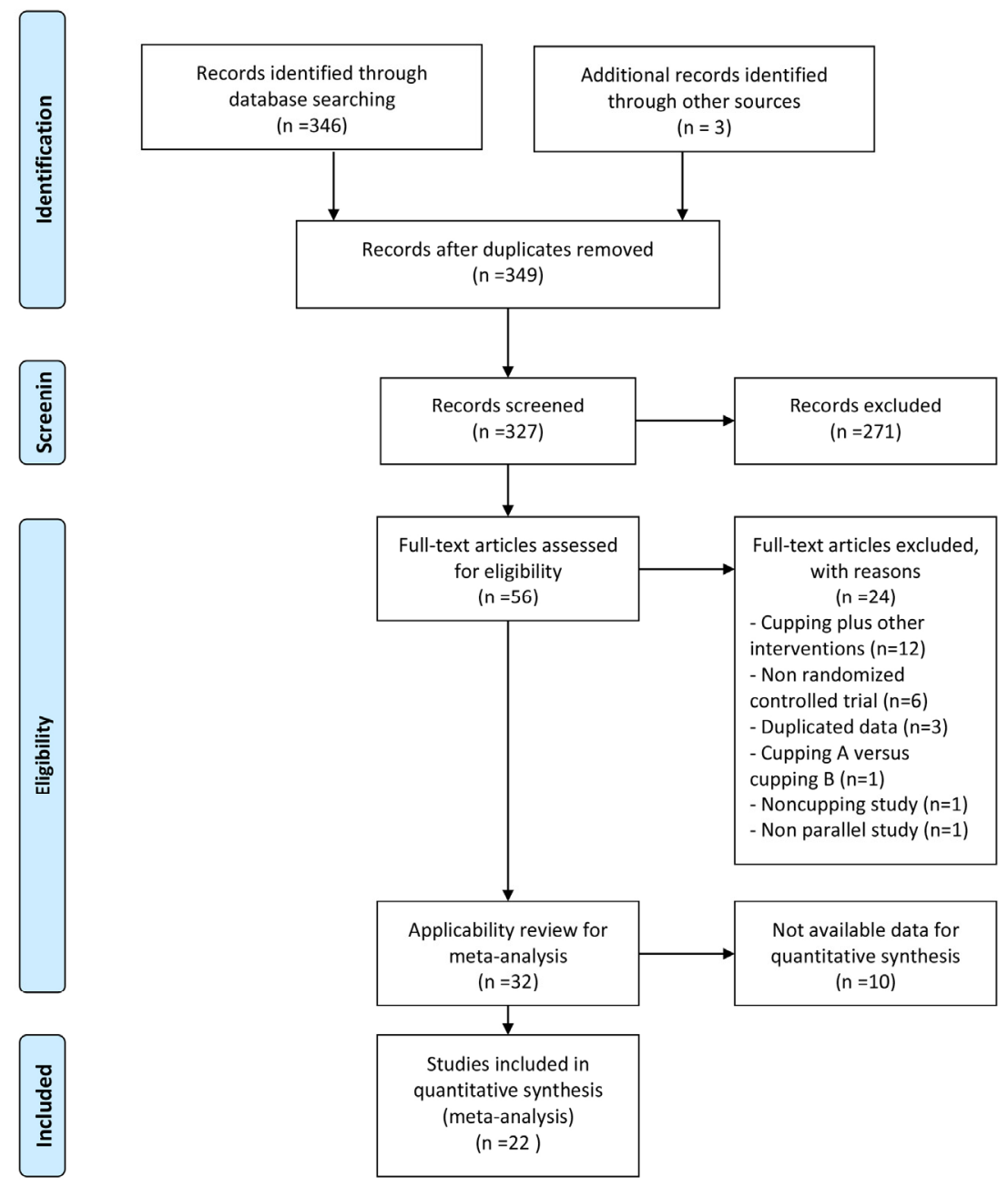

Fig. 1. Flowchart of the study selection

A total of 374 articles were identified through the database search, and 3 articles were identified through hand searching, and of these, 50 were excluded due to duplication. The titles and abstracts of the remaining 327 articles were screened, and 271 articles not suitable for this study were excluded. Full texts of the remaining 56 articles were read and 24 articles that did not meet the eligibility criteria of this study were excluded.
Out of the remaining 32 articles, 22 articles providing data suitable for quantitative synthesis were finally selected for meta-analysis and methodological quality assessment. The study selection process is summarized in Fig. 1, and a list of articles excluded during the process from full text reading to the final selection is presented in Supplement 4. 


\section{Characteristics of the included studies}

1) Study design and sample size

A total of 22 studies $^{11-32)}$ with 1653 participants published in China from 2001 to 2020 were included in the final analysis. Most of them were studies with a 2-arm design with the exception of three studies ${ }^{14,19,31)}$ with 3 arms. The number of participants included in the posttreatment evaluation ranged from 40 to 135 (Table 1).

\section{2) Participants}

The participants of all included studies were patients with stroke, and none of the studies limited the stroke subtype of the patients. There was no study that recruited only patients in the acute stage within one week of stroke onset, but most of the participants were patients within 6 months of stroke onset. Of the exceptions, 4 studies included patients with more than 6 months since stroke onset, ${ }^{15,18,30,31)}$ and 3 studies did not provide any information regarding the time from onset (Table 1) $)^{22,23,32)}$.

The most commonly treated stroke condition was spasticity, which was reported in 9 studies $12-14,16,17,20,23,24,31)$. Six ${ }^{12-14,16,17,24)}$ of these studies targeted patients with upper limb spasticity, of which $4^{12,14,16,17)}$ were about elbow spasticity. There were three studies each on spasticity in lower $\operatorname{limbs}^{23)}$, four limbs, ${ }^{31)}$ and nonspecified parts $^{20}$. The most commonly studied stroke conditions after spasticity were hemiplegic shoulder pain ( 5 studies) ${ }^{19,22,25,26,28)}$ and poststroke shoulder-hand syndrome (4 studies). ${ }^{21,27,29,30)}$ The remaining studies targeted sensory dysfunction ${ }^{18,32)}$, upper limb dysfunction $^{15)}$, and neglect syndrome (Table 1) ${ }^{11)}$.

Two studies considered the type of syndrome differentiation based on traditional Chinese medicine. ${ }^{1520)}$ One enrolled patients with spasticity and qi deficiency with blood stasis type ${ }^{15)}$ and the other enrolled patients with upper limb dysfunction with phlegm and blood stasis obstructing collaterals type (Table 1$)^{20)}$.

3) Intervention, control and outcome measures

(1) A versus $B$ type studies

Two studies ${ }^{1724}$ compared the effects of wet cupping and Western medication (baclofen, a muscle relaxant) in patients with upper limb spasticity. In both studies, the treatment period was 4 weeks, the treatment group received wet cupping on the center of the biceps brachii belly twice a week, and the control group was orally administered $10 \mathrm{mg}$ of baclofen three times a day. These two studies evaluated changes in upper limb motor function and spasticity. In terms of the safety of the cupping therapy, one study ${ }^{24}$ described the plan for adverse event monitoring in the methods session but did not report the results. Another study ${ }^{17)}$ did not provide any information on the safety of cupping therapy (Table $1 \& 2$ ).

\section{(2) $\mathrm{A}+\mathrm{B}$ versus $\mathrm{B}$ type studies}

Twenty studies ${ }^{11-16,18-23,25-32)}$ were designed as an $\mathrm{A}+\mathrm{B}$ versus $\mathrm{B}$ form, comparing the add-on effect of cupping therapy with the noncupping control in patients receiving standard treatment for stroke. Three of them ${ }^{14,19,23)}$ were 3 -arm studies, and the other 18 were 2-arm studies. No studies used sham devices or placebo controls (Table 1).

Of the 20 studies, $5^{11,19,21,25,31)}$ used dry cupping and $15^{12-16,18,20,22,23,26-30,32)}$ used wet cupping. The 
retention time for cupping was between 5 and 15 minutes in both dry and wet cupping studies. The amount of blood drawn by wet cupping therapy ranged from a few drops to $10 \mathrm{ml}$. Ten ${ }^{13,15,18-20,22,}$ 25-27,32) of the included studies performed cupping therapy once every other day. The next most common protocols were once a day ${ }^{14,28,30,31)}$ and twice a week ${ }^{12,16,23)}$. The treatment period was most frequently 4 weeks $^{11,12,16,18,21)}$ and ranged between at least 2 weeks and at most 6 months. The most common sites for cupping were the pain site of the affected shoulder for patients with hemiplegic shoulder pain or shoulder-hand syndrome and the center of the biceps brachii belly for patients with upper limb spasticity (Table 1).

The therapeutic effects of cupping therapy for stroke patients was evaluated on various scales. Most of the studies presented the response rates for the main outcomes in stroke survivors. In addition, many other studies measured upper limb motor function using the Fugl-Meyer assessment (FMA) scale, and there were also studies that evaluated overall motor function or sensory function. Some studies assessed the intensity of shoulder pain using a visual analog scale (VAS) or numeric rating scale. There were also studies that assessed the activities of daily living using the Barthel or modified Barthel index. There was only one study ${ }^{15)}$ that assessed neurological deficits using an internationally accepted stroke scale, such as the National Institute Health stroke scale, and no study reported mortality, disability, or recurrence of stroke (Table $1 \& 2$ ).

Most studies did not mention safety. One study on dry cupping ${ }^{21)}$ reported that any serious adverse events were observed, and three studies on wet cupping ${ }^{13,15,25)}$ described the adverse events identified during the study period (Table 1 \& 2).

\section{RoB of the included studies}

Only two studies ${ }^{15,24)}$, which provided enough information to confirm that both the random sequence generation and allocation concealment process were properly performed, met the first domain of the RoB 2 (randomization process). The remaining included studies did not provide enough information to judge relevant procedures and were judged to be at the level of raising some concerns in this domain.

In the second domain, deviations from the intended interventions, all studies were judged to be at low RoB. Although patient blinding and performer blinding were not performed, it was judged that this situation was due to the unique nature of the cupping therapy and would not have affected the intended intervention.

In the third domain, missing outcome data, most studies were judged to be at high RoB. This was because they did not mention anything about loss to follow-up or dropout and we could not rule out the possibility that any missingness led to biased results. Only 3 studies $^{15,18,24)}$ reported dropout, and we could confirm that the missingness did not bias the results, these studies were judged to be at low RoB.

In the fourth domain, measurement of the outcome, all the included studies were judged to be at high RoB. This was because no studies mentioned anything about the achievement or impact of assessor blinding. 
(518) Journal of Korean Medicine 2021;42(4)

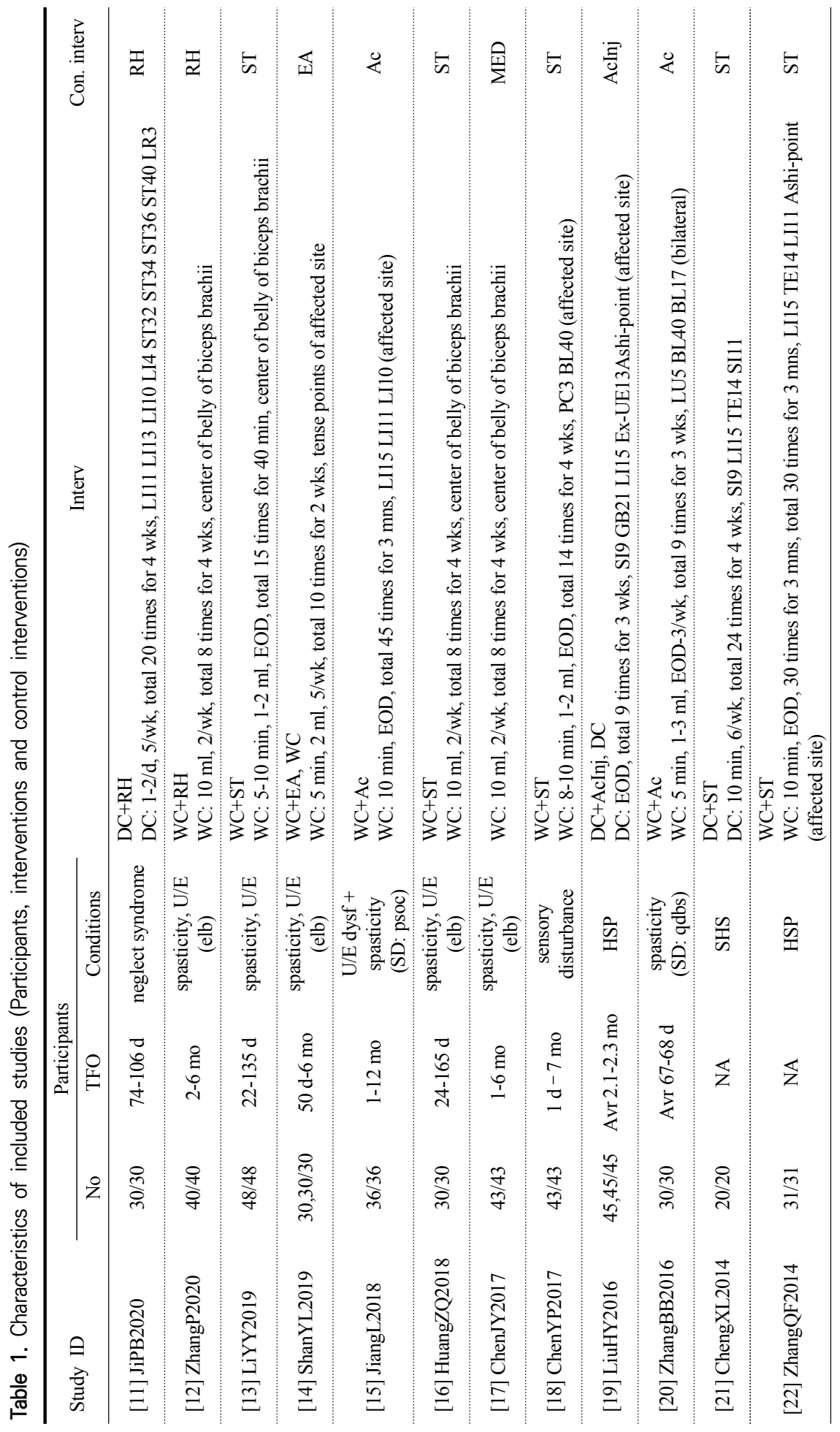




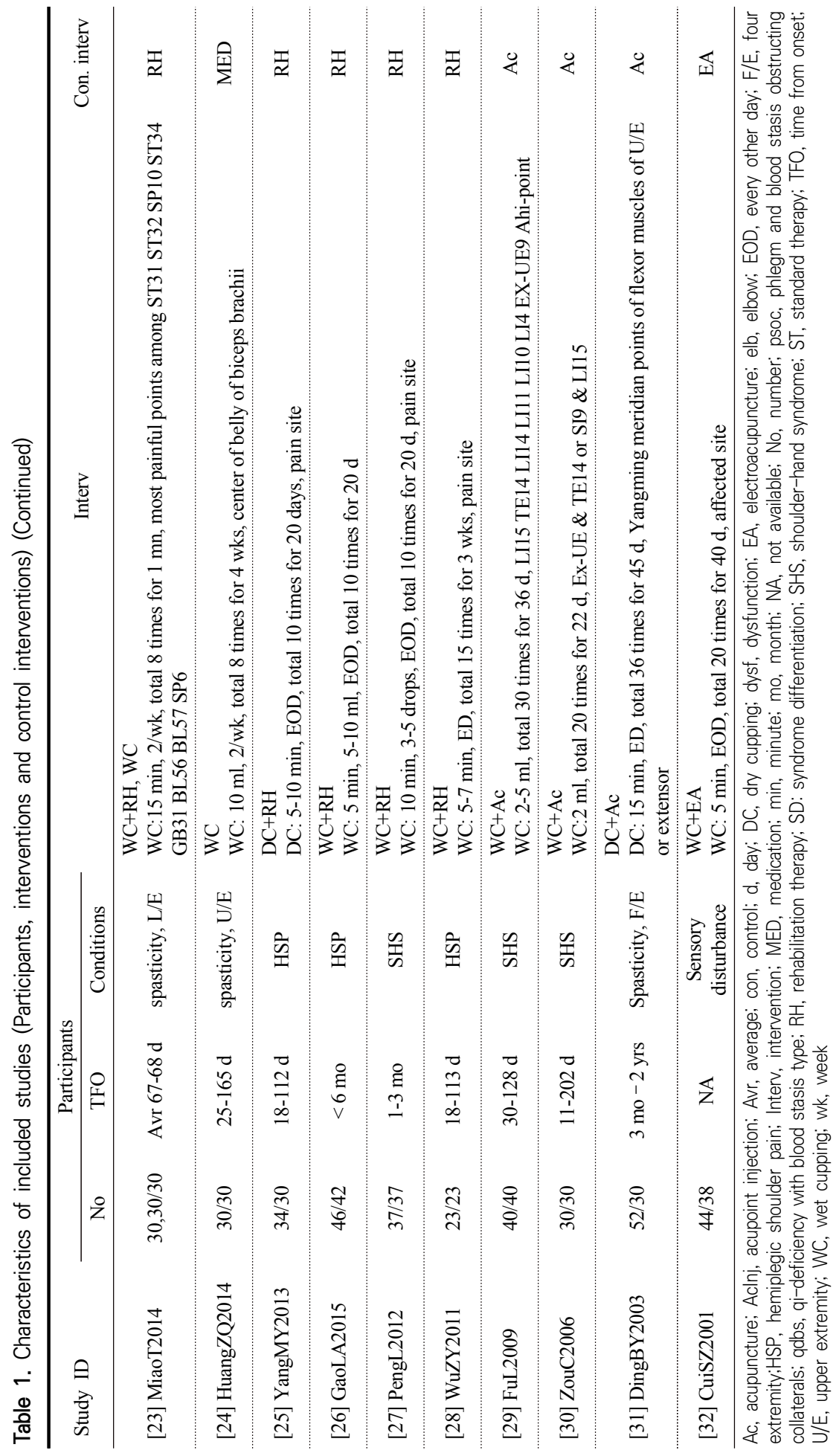




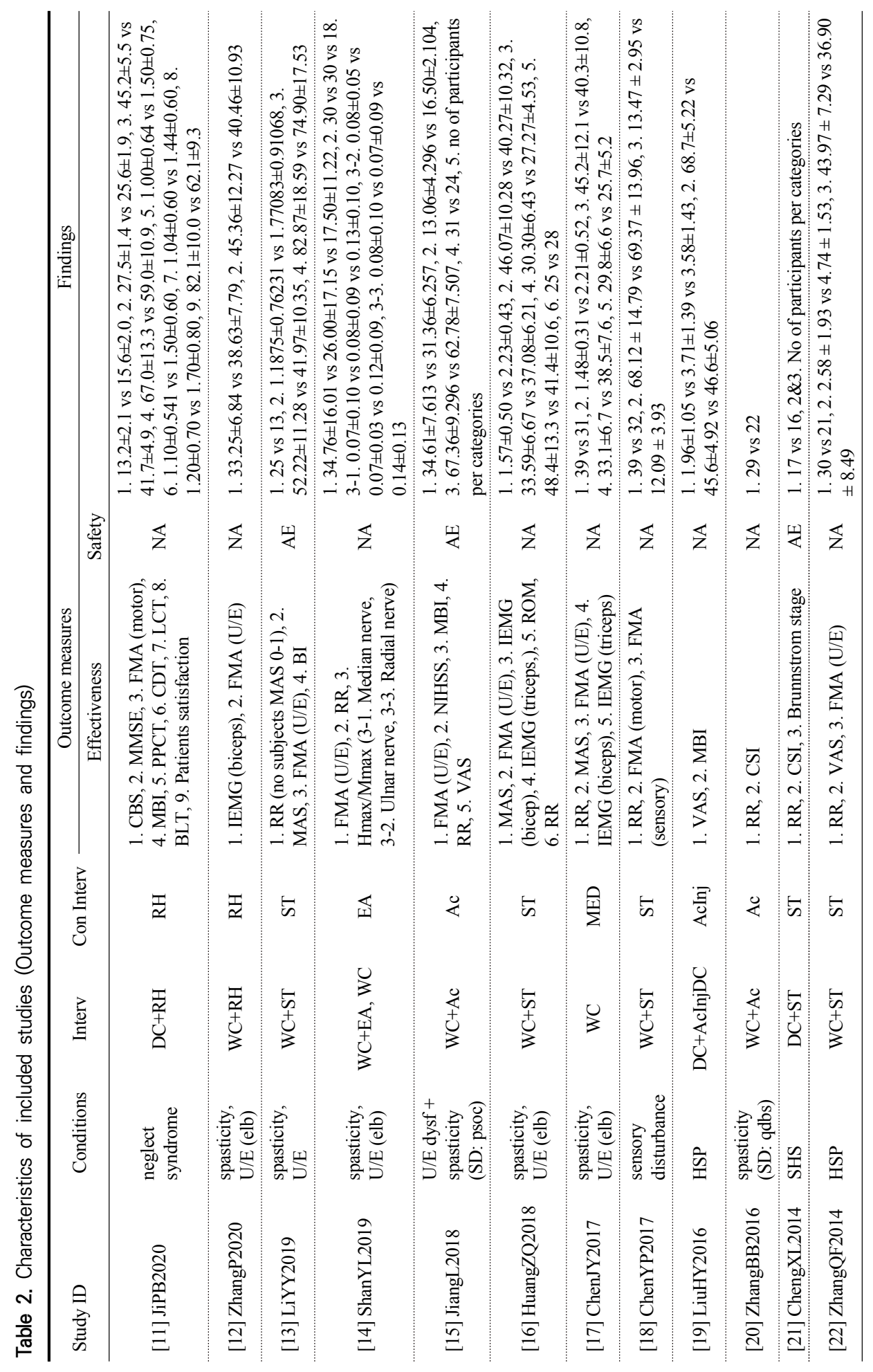




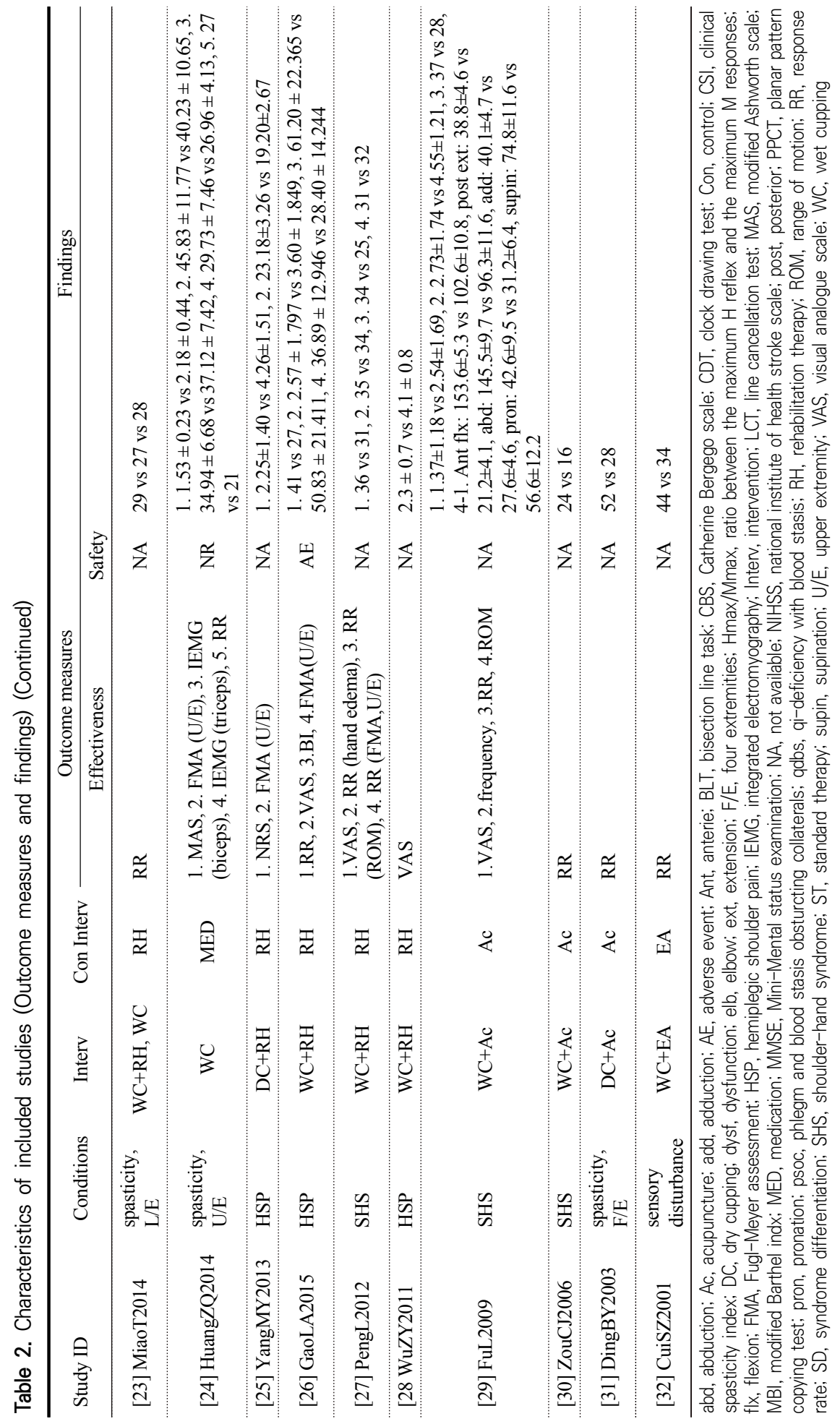




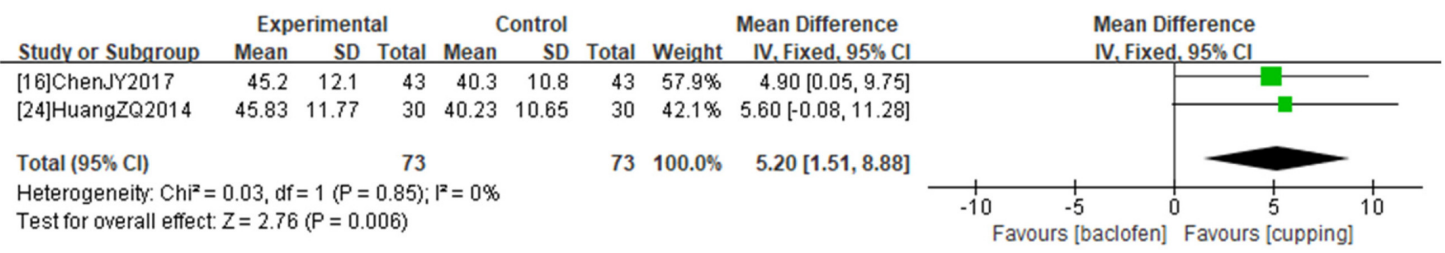

Fig. 2. Forest plot of the effect of cupping therapy versus medication on upper limb motor function Assessed by upper limb motor function subdomain of the Fugl-Meyer assessment scale

In the fifth domain, selection of the reported result, we judged that some concerns were raised in the RoB of all included studies. No studies involved previously published study protocol papers prior to initiation of trial. Therefore, we reviewed whether the results session was described in accordance with the plans mentioned in the Methods section of the paper. No significant biases were identified in the Methods and Result sections. However, no studies provided information that could confirm whether the reported outcome was selected from among multiple outcome measurements, and therefore, this issue raised some concerns for all included studies.

The overall RoB of all included studies derived by synthesizing the evaluations in the above domains was judged to be high. The results of the RoB assessment for each domain of the included studies are schematically shown in Supplements 5 and 6.

\section{Meta-analysis and evidence level}

1) Effectiveness of cupping therapy compared to Western medication

Two studies ${ }^{1724)}$ compared the effect of wet cupping therapy with Western medication (baclofen $10 \mathrm{mg}$ tid pc [P.O.]) in patients with upper limb spasticity after stroke. The meta-analysis of these two studies showed that cupping therapy was significantly more advantageous in improving upper limb dysfunction assessed by the subdomain score of the FMA scale (MD 5.20, 95\% CI 1.51 to $8.88, \mathrm{P}=0.006$ ) (Fig. 2). The degree of spasticity assessed by the modified Ashworth scale (MAS) was also significantly improved in the cupping therapy group compared to the Western medication group (MD $-0.69,95 \% \mathrm{CI}-0.82$ to $-0.56, \mathrm{P}<0.00001$ ) (Fig. 3(A)). The proportion of patients who responded positively regarding the clinical aspects of spasticity was also significantly higher in the cupping group (MD 3.81, 95\% CI 1.50 to 9.65 , $\mathrm{P}=0.005$ ) (Fig. 3(B)). Changes in the biceps and triceps muscles detected by integrated electromyography were also more favorable with cupping therapy (MD $-4.05,95 \%$ CI -6.36 to -1.74, $\mathrm{P}=0.0006 ; \mathrm{MD}=3.56,95 \%$ CI 1.62 to 5.50 , $\mathrm{P}=0.0003$ ) (Fig. $3(\mathrm{C}, \mathrm{D})$ ). The certainty of the evidence assessed by the GRADE approach was low in all cases. This was because of the high RoB and small sample size of the included studies (Supplement 7).

\section{2) Effectiveness of cupping therapy as an add-on to conventional treatment \\ (1) Activities of daily living (ADL)}

Five studies ${ }^{11,13,15,19,26)}$ assessed the additive 


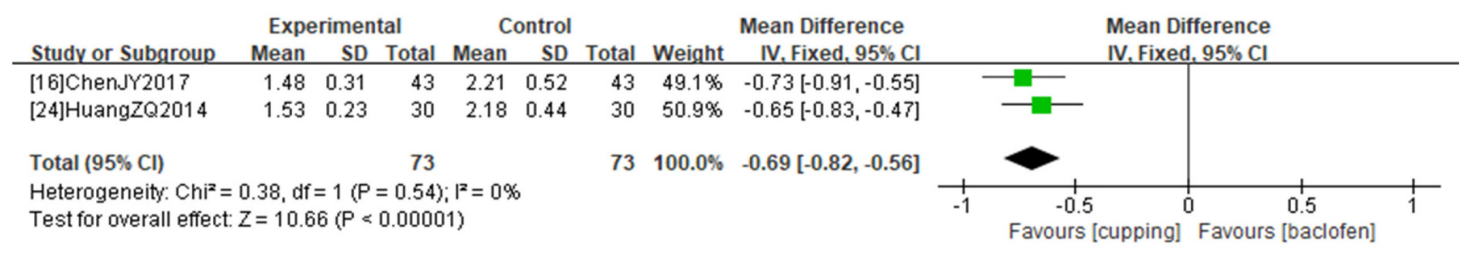

(A) Modified Ashworth scale

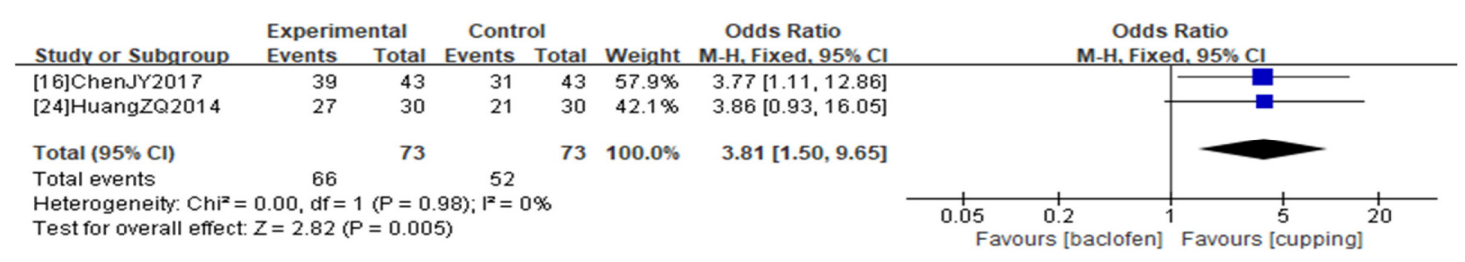

(B) Response rate

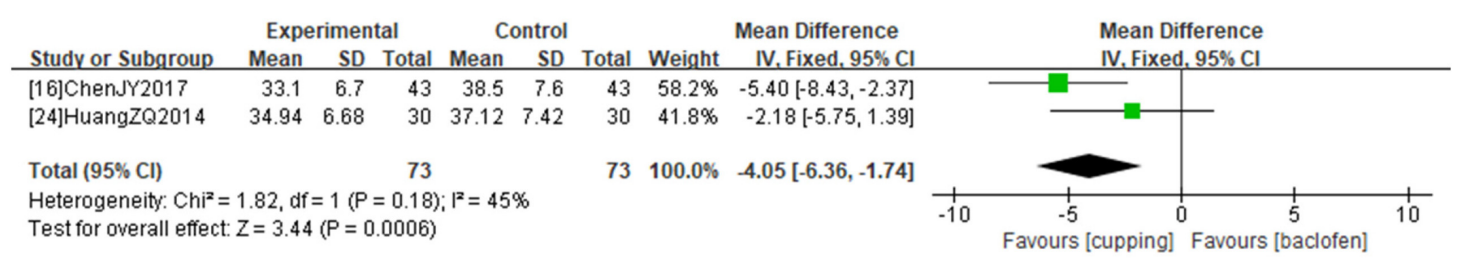

(C) Integrated electromyography (biceps brachii)

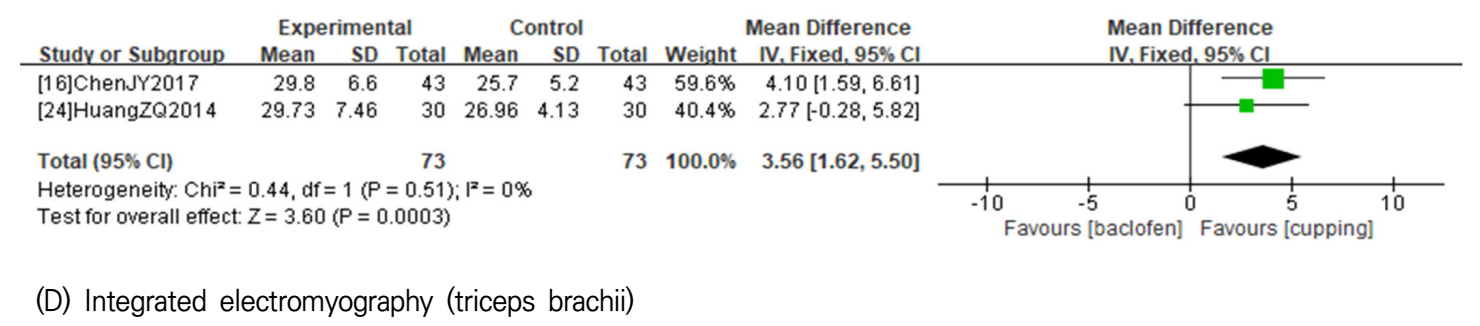

Fig. 3. Forest plot of the effect of cupping therapy versus medication on spasticity

effect of cupping therapy using the Barthel index (BI) or modified Barthel index (MBI). Combining the data, the additional implementation of cupping therapy for patients who were receiving conventional treatment was significantly more advantageous than the noncupping condition (SMD 1.23, 95\% CI 0.21 to $2.25, \mathrm{P}=0.02$ ) (Fig. 4), and the level of evidence supporting this result was low (Supplement 8). The subgroup analysis including only wet cupping studies ${ }^{13,15,26)}$ showed similar results (SMD 0.48, 95\% CI 0.23 to 0.72 , $\mathrm{P}=0.0002$ ) (Fig. 4). The heterogeneity detected in the whole synthesis $\left(\mathrm{I}^{2}=95 \%\right)$ was resolved in the wet cupping subgroup analysis $\left(\mathrm{I}^{2}=0 \%\right.$ ) (Fig. 4). 


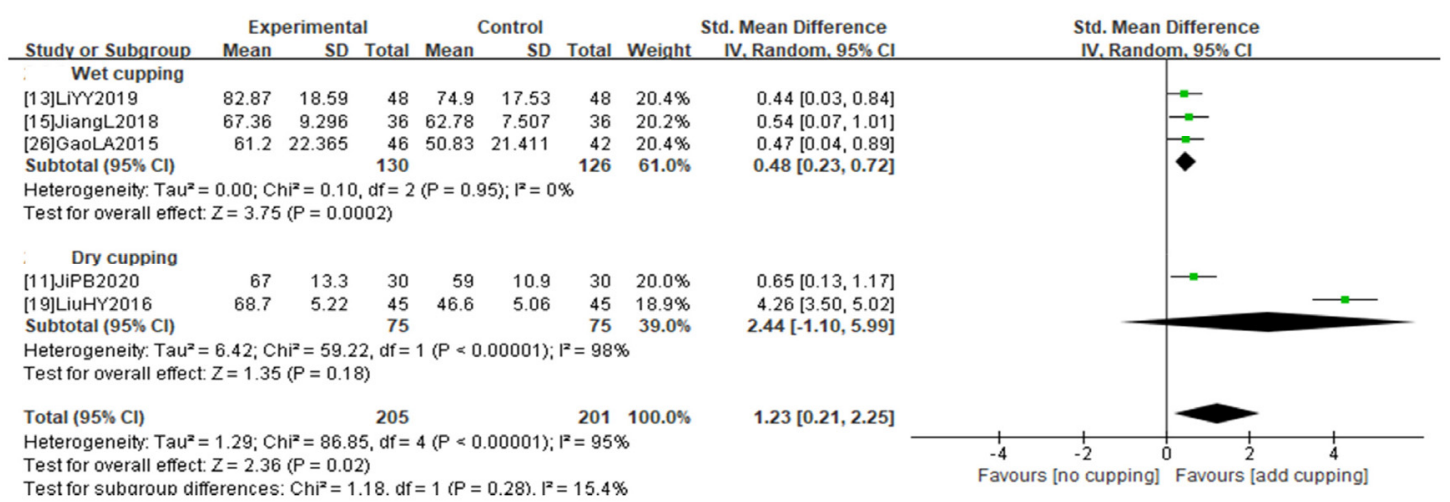

Fig. 4. Forest plot of the effect of additional cupping therapy on activities of daily living Assessed by Barthel index

However, when pooling the two studies involving only dry cupping ${ }^{11,19)}$, the significant benefit of cupping therapy disappeared (SMD 2.44, 95\% CI -1.10 to $5.99, \mathrm{P}=0.18$ ) (Fig. 4), and the level of evidence was also downgraded (Supplement 8).

\section{(2) Motor function}

i. General motor function

Only wet cupping study ${ }^{18)}$ and one dry cupping study ${ }^{11)}$ evaluated the change in the motor function subdomain score on the FMA scale. Only the dry cupping study showed a significant benefit of cupping therapy (MD 3.50, 95\% CI 0.86 to $6.14, \mathrm{P}=0.009$ ) (Fig. 5(A)). However, when combining the two studies, the additional benefits of cupping therapy were not confirmed (MD 1.95, 95\% CI -2.42 to $6.31, \mathrm{P}=0.38$ ) (Fig. $5(\mathrm{~A})$ ), and the level of evidence was still very low (Supplement 8).

\section{ii. Upper limb motor function}

Seven studies using wet cupping ${ }^{12-16,22,26)}$ and one study using dry cupping ${ }^{25)}$ reported changes in the upper limb motor function subdomain score of the FMA scale. All studies showed significantly beneficial effects of additive cupping therapy (Fig. 5(B)). Meta-analysis also showed positive results of cupping therapy (MD 6.91, 95\% CI 4.36 to $9.46, \mathrm{P}<0.00001$ ) (Fig. 5(B)), which was supported by a moderate level of evidence (Supplement 6). The subgroup analysis including only wet cupping studies showed similar results (MD 7.66, 95\% CI 4.64 to 10.67, $\mathrm{P}=0.01$ ) (Fig. 5(B)) (Supplement 8).

iii. Sensory function

Two studies ${ }^{18,32)}$ compared the response rates with and without additional wet cupping therapy in patients with sensory impairment after stroke. Meta-analysis of these studies showed that the benefit of wet cupping therapy was significant and the level of evidence supporting the result was low (OR 4.35, 95\% CI 1.42 to 13.32, $\mathrm{P}=0.010$ ) (Fig. 6) (Supplement 8).

\section{iv. Spasticity}

Two studies ${ }^{13,16)}$ assessed the level of spasticity using the MAS before and after wet cupping 


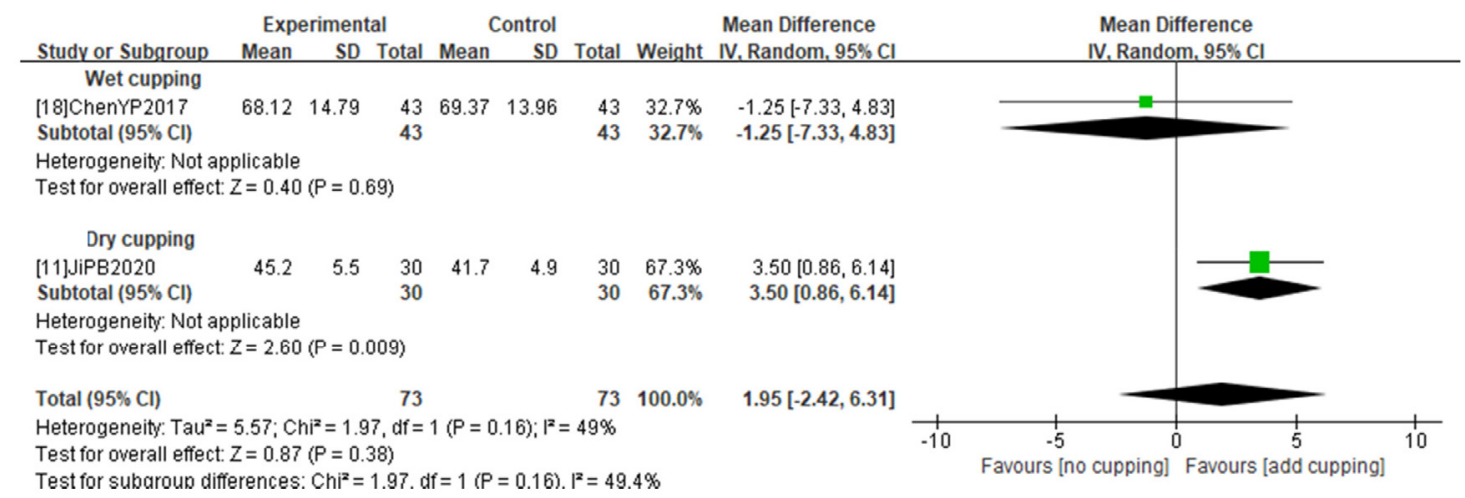

(A) General motor function (motor function subdomain of Fugl-Meyer assessment scale)

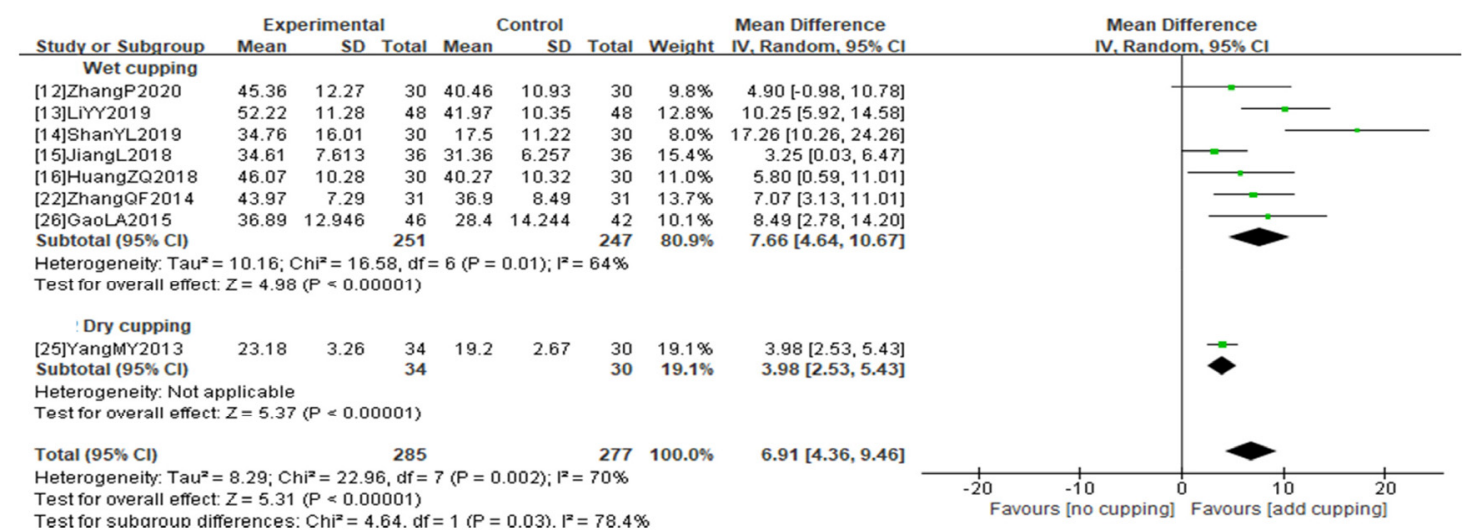

(B) Upper limb motor function (upper limb motor function subdomain of Fugl-Meyer assessment scale)

Fig. 5. Forest plot of the effect of additional cupping therapy on motor function

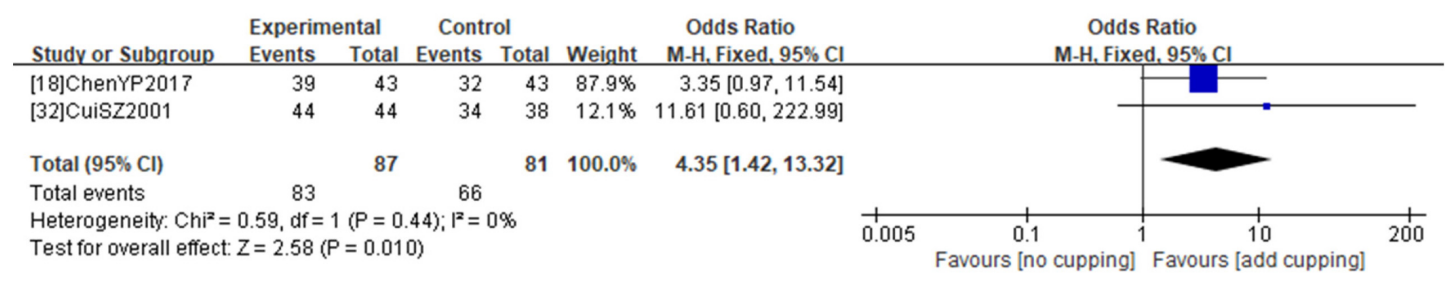

Fig. 6. Forest plot of the effect of additional cupping therapy on sensory function Assessed by sensory domain of Fugl-Meyer assessment scale

therapy in patients with upper arm spasticity. The meta-analysis of these two studies showed that the additional effect of wet cupping was significant with a low level of evidence (MD
$-0.63,95 \%$ CI -0.83 to $-0.44, \mathrm{P}<0.00001$ ) (Fig. 7(A)) (Supplement 8).

Seven studies ${ }^{13-15,20,23,31)}$ investigated the proportion of participants who responded positively in terms 


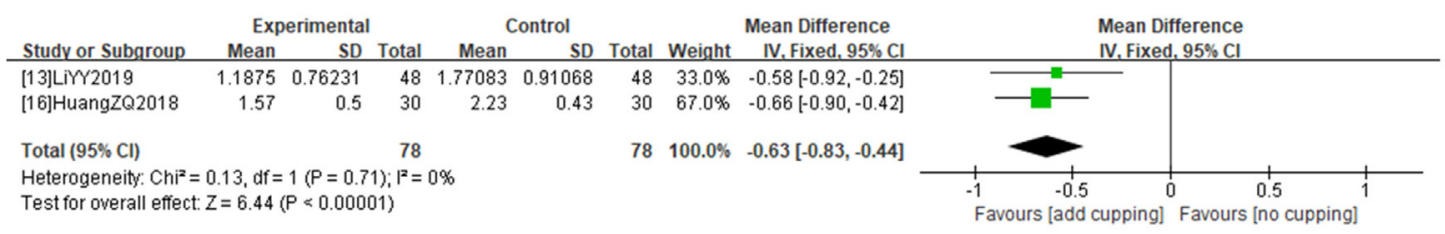

(A) Modified Ashworth scale

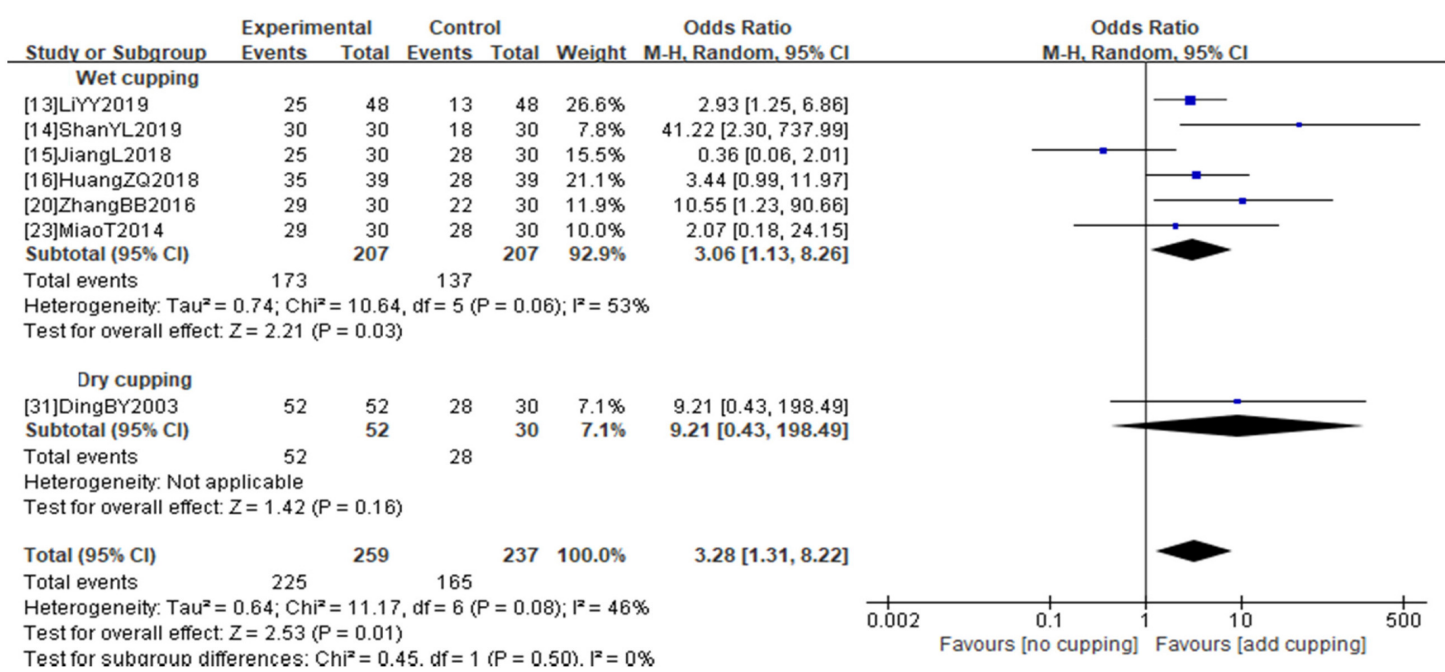

(B) Response rate

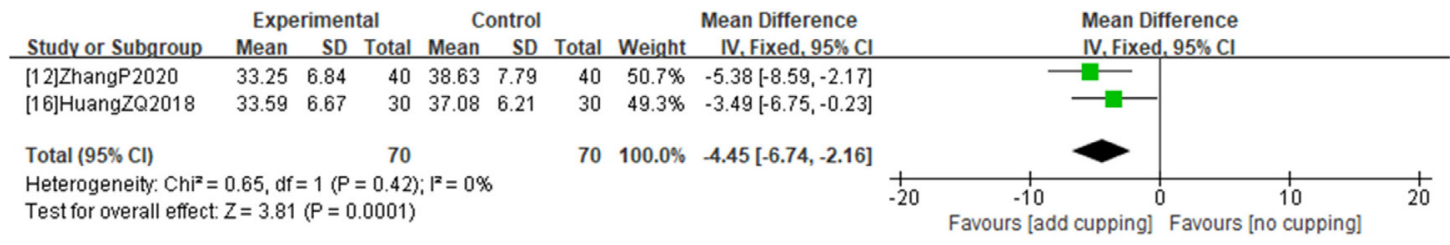

(C) Integrated electromyography (biceps brachii)

Fig. 7. Forest plot of the effect of additional cupping therapy on spasticity

of spasticity after cupping therapy. The meta -analysis of these studies showed the significant benefit of cupping therapy (OR 3.28, 95\% CI 1.31 to $8.22, \mathrm{P}=0.01$ ) (Fig. 7(B)), and a moderate level of evidence supported this result (Supplement 8). The subgroup analysis of the wet cupping studies showed similar results (OR 3.06, 95\% CI 1.13 to $8.26, \mathrm{P}=0.03$ ), whereas the dry cupping subgroup did not (OR 9.21, 95\% CI 0.43 to 198.49, $\mathrm{P}=0.16$ ) (Fig. 7(B)) (Supplement 8).

Changes in biceps brachii muscle detected by integrated electromyography in patients with upper limb spasticity were also more favorable for wet cupping (MD $-4.45,95 \%$ CI -6.74 to -2.16, $\mathrm{P}=0.0001) \quad(\text { Fig. } 7(\mathrm{C}))^{12,16}$. However, the level of evidence supporting this benefit was very 


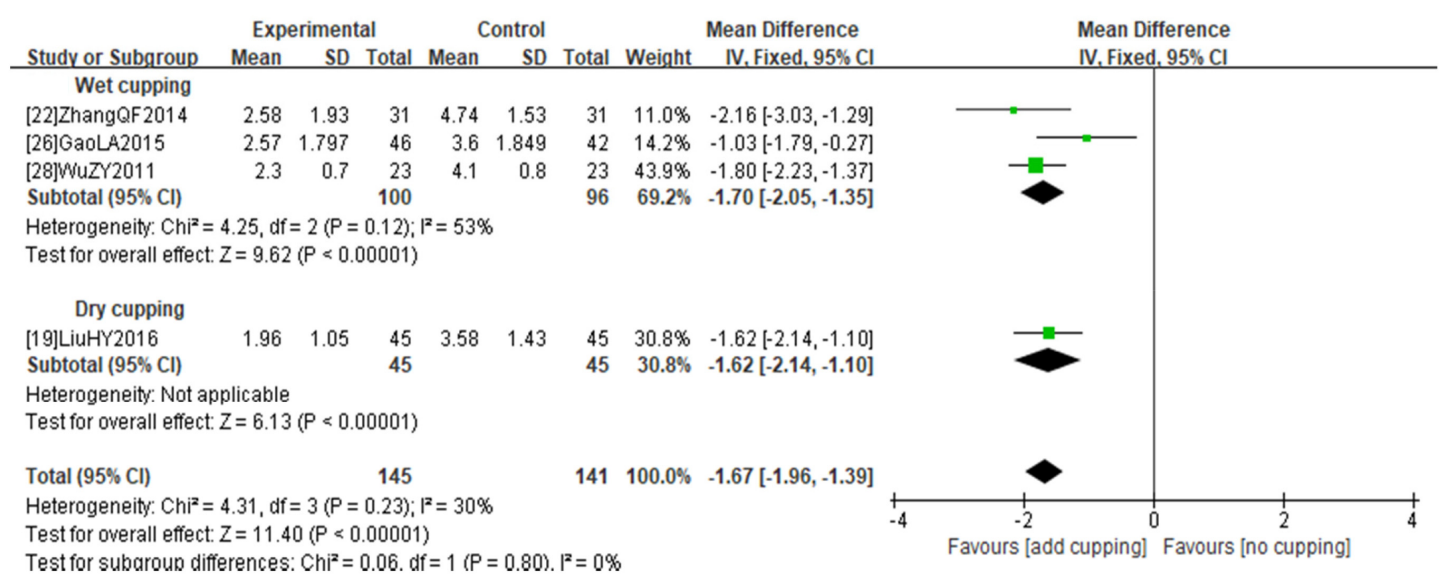

(A) Visual analog scale

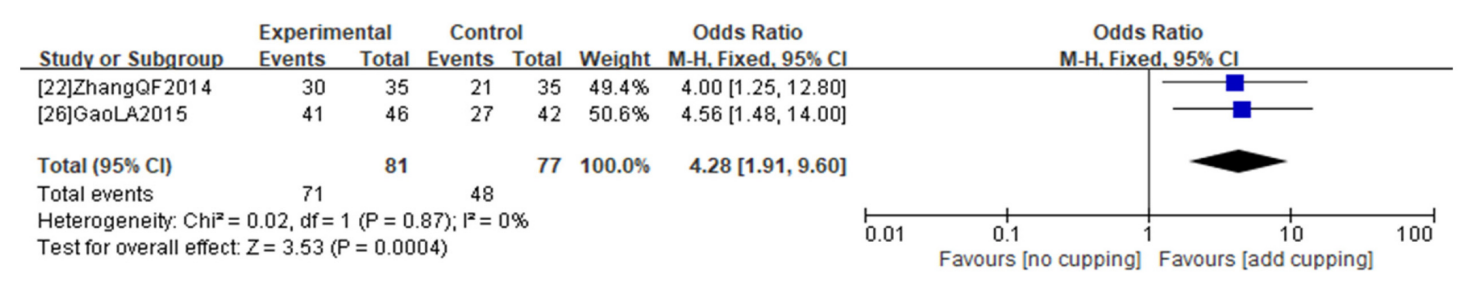

(B) Response rate

Fig. 8. Forest plot of the effect of additional cupping therapy on shoulder pain

low (Supplement 8).

v. Shoulder pain

Four studies ${ }^{19,22,26,28)}$ reported changes in shoulder pain measured by a VAS after additive cupping therapy for patients with hemiplegic shoulder pain. The meta-analysis of these studies showed that adjuvant cupping therapy had significant benefits in reducing VAS scores (MD $-1.67,95 \%$ CI -1.96 to $-1.39, \mathrm{P}<0.00001$ ) (Fig. $8(\mathrm{~A})$ ), although the level of evidence supporting this effect was very low (Supplement 6). Similar results were reproduced in the subgroup analysis including only the wet cupping studies (MD -1.70, 95\% CI -2.05 to -1.35 ) (Fig. 8(A)) (Supplement 8).
Two studies ${ }^{22,26)}$ investigated the proportion of hemiplegic shoulder pain patients who responded positively to wet cupping therapy. Combining these studies showed that additive wet cupping therapy had significant benefits in improving shoulder pain, although the level of evidence was very low (OR 4.28, 95\% CI 1.91 to 9.60, $\mathrm{P}=0.0004$ ) (Fig. 8(B)) (Supplement 8).

vi. Shoulder-hand syndrome

Four studies ${ }^{21,27,29,30)}$ assessed the response rates to cupping therapy for patients with poststroke shoulder-hand syndrome. The meta-analysis of these studies showed that cupping therapy had significant benefits in increasing the response rate 


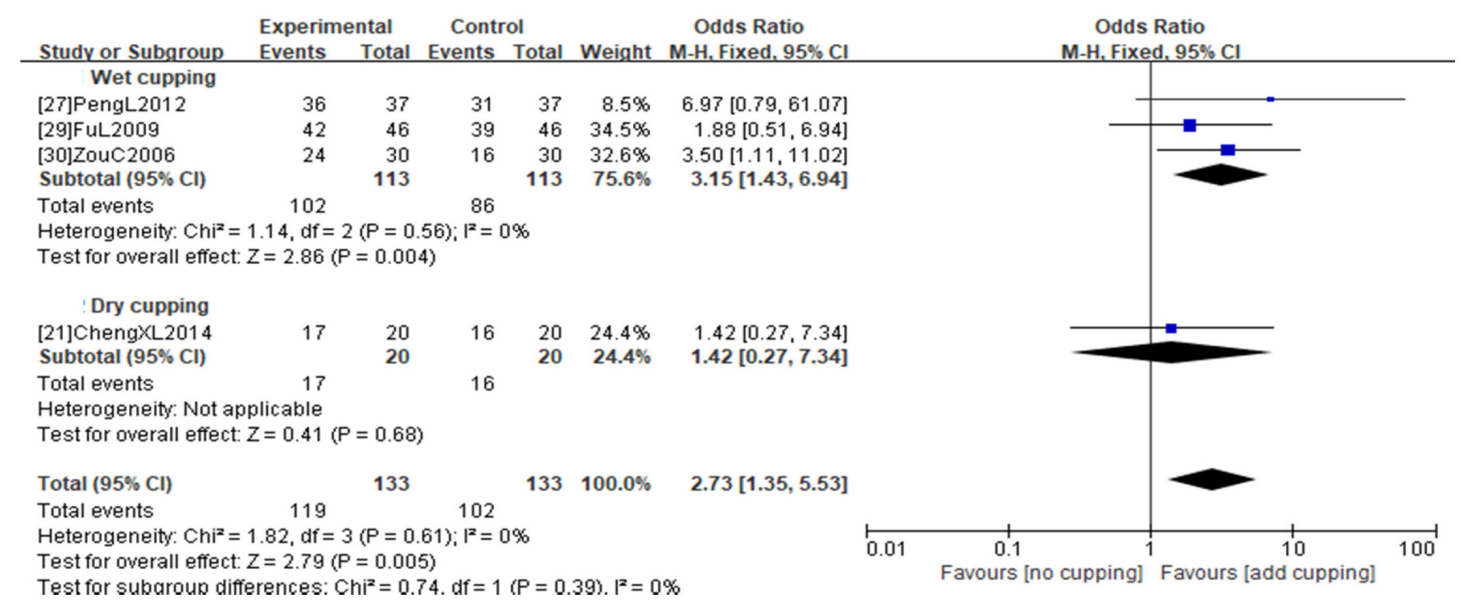

Fig. 9. Forest plot of the effect of additional cupping therapy on shoulder-hand syndrome Assessed by response rate

in patients with poststroke shoulder-hand syndrome (OR 2.73, 95\% CI 1.35 to 5.53, $\mathrm{P}=0.005$ ) (Fig. 9). The results of the subgroup analysis of studies involving only wet cupping therapy also reproduced similar results (OR 3.15, 95\% CI 1.43 to 6.94, $\mathrm{P}=0.004$ ) (Fig. 9). However, the level of evidence supporting these findings was very low in all cases (Supplement 8).

\section{vii. Safety}

Five studies ${ }^{13,15,21,24,26)}$ mentioned plans for collecting adverse events (AEs) during the study period. All of them were designed to compare the additional effectiveness of cupping therapy with the noncupping control. One ${ }^{24)}$ of the five studies mentioned the AE collection plan in the Methods section of the article but did not provide any information on the safety profile in the Result section. Out of the 4 studies describing the intervention-related AEs in the Results section, one study $^{21)}$ was about dry cupping, and the others $^{13,15,26)}$ were about wet cupping. The dry cupping study ${ }^{21)}$ reported that they did not identify any serious AEs in either cupping or noncupping groups. One of the wet cupping studies reported the occurrence of blistering with pain after cupping therapy, but did not disclose how many cases were identified ${ }^{26)}$. Therefore, we could secure data available for meta-analysis from only two wet cupping studies ${ }^{13,15)}$.

One study provided wet cupping therapy every other day for 30 days to patients with upper arm spasticity and reported two cases of subcutaneous bleeding at the cupping site ${ }^{13)}$. These AEs spontaneously disappeared after 1 week, and no AEs were identified in the control group ${ }^{13)}$. Another study provided wet cupping therapy every other day for 3 months to patients with upper arm dysfunction and identified two cases of AEs, including either bleeding or pain ${ }^{15}$. Combining the AEs identified in these two studies, no significant difference was found in the occurrence of AEs between the additional cupping group and the noncupping control group (OR 6.33, 95\% CI 


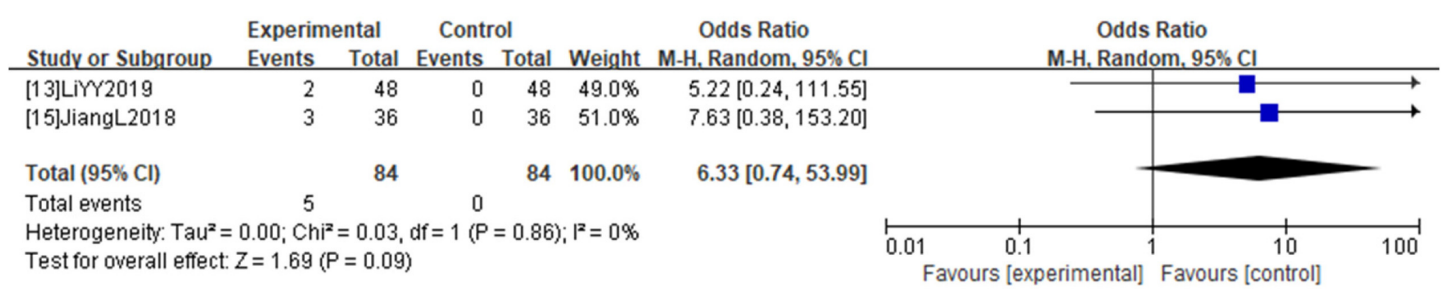

Fig. 10. Forest plot of the safety of additional cupping therapy Assessed by incidence of adverse events

0.74 to $53.99, \mathrm{P}=0.09$ ) (Fig. 10), and the level of evidence supporting this result was very low (Supplement 8).

\section{Discussion}

To confirm the therapeutic effect of cupping therapy, one of the oldest interventions used across the world in multiple cultures ${ }^{5)}$, scientific explorations have been attempted in modern times. Clinical trials have shown the potential of cupping therapy to be effective in a variety of diseases, including herpes zoster, facial palsy, acne, and cervical spondylosis, as well as pain ${ }^{33}$, 34).

Nevertheless, the mechanism of action of cupping remains unclear ${ }^{35,36)}$. However, the hypotheses that this intervention has local effects, including improving capillary and lymphatic circulation and extension of the underlying tissue, as well as systemic effects through activation of the neuroendocrine immune system resulting from the signaling molecules released from the blood vessels or tissues damaged by the cupping procedure $^{35,36)}$, support the reason why cupping therapy can be applied to a variety of conditions across multiple systems of the whole body.

Among the wide range of indications for cupping therapy, this study mainly presented the effects related to improving various complications in stroke survivors. This topic had already been addressed by a previous systematic review published in $2010^{6}$. However, at the time, sufficient data for meta-analysis were not obtained, so only narrative reviews of five clinical studies were possible $^{6)}$. Since that previous review ${ }^{6)}$, more RCTs exploring the effect of cupping therapy in patients with stroke have been published. Therefore, we updated the findings of the previous study ${ }^{6}$ by performing quantitative synthesis and qualitative evaluation of the currently available evidence. To the best of our knowledge, this study is the first to systematically review and meta-analyze RCTs on the effectiveness and safety of cupping therapy in stroke survivors.

The findings of this study showed the potential of cupping therapy as an add-on intervention for stroke survivors receiving conventional treatment. Among the various clinical outcomes, the additional benefits of wet cupping therapy for improving motor function of the upper limb were identified. Cupping therapy may have directly influenced upper limb motor function. However, considering that the original studies supporting this result were derived from patients with upper limb spasticity or hemiplegic shoulder pain and 
that the main findings of this systematic review also showed the advantage of cupping therapy for improving upper limb spasticity and reducing shoulder pain, we speculate that the effect of wet cupping for improving upper limb motor function may have been the result of muscle tone control and pain relief effects. However, this study could not find evidence supporting the effect of cupping therapy on lower limb motor function.

Another finding of this study, supported by a moderate level of evidence, was that additional wet cupping therapy increased the positive response rate for poststroke spasticity. This effect of wet cupping therapy, although at a very low evidence level, was also confirmed by improvements in MAS scores and decreases in muscle tone of the affected biceps brachii, a flexor muscle that is generally overhypertensive in poststroke elbow spasticity. These findings seem to reflect the possibility that muscle relaxation, one of the known actions of cupping therapy ${ }^{37)}$, could also work on upper limb spasticity in stroke patients.

Other studies have shown the positive potential of adjuvant cupping therapy in a wider range of indications. This study identified additional benefits of cupping therapy for improving ADLs, sensory dysfunction, shoulder pain, and shoulder -hand syndrome in stroke survivors, although the level of supportive evidence was low or very low. The effect of additional cupping to reduce pain and improve functions was confirmed in previous studies targeting patients with pain in various underlying diseases ${ }^{4,5)}$, and this study confirmed that this therapeutic action of cupping therapy could also work in stroke survivors.
This study also identified head-to-head studies comparing the effects of wet cupping therapy with active control interventions. The meta-analysis of these studies showed the possibility of wet cupping therapy being more beneficial than oral administration of baclofen in improving muscle tone and motor function in patients with poststroke upper arm spasticity. However, since there were only two small-sized studies supporting the findings and the level of evidence was low, it is necessary to be careful in interpreting this result.

The findings of this study mainly supported the additional benefits of cupping therapy, especially wet cupping therapy, in upper limb motor function, upper limb spasticity, shoulder pain and shoulder-hand syndrome in stroke survivors. Although the level of evidence was often low or very low, with rare studies with moderate levels of evidence, these findings may be helpful in determining priorities when considering application of cupping therapy for stroke survivors.

Subgroup analysis of wet cupping or dry cupping studies showed that the number of wet cupping studies was higher, and the treatment effect and evidence level were also higher than those of dry cupping studies. The fact that there were more studies on wet cupping than on dry cupping is consistent with the results of previous studies ${ }^{6,33)}$. We found that not only was the number of studies on dry cupping therapy small, but the heterogeneity between those studies was also great. Therefore, it is difficult to draw conclusions about the effectiveness of dry cupping therapy on stroke based on the findings of this study. More clinical evidence needs to be collected. 
Further consideration is also required on the effects of wet cupping therapy on stroke survivors, considering that wet cupping is a complex form of at least two kinds of interventions, namely, cupping and bloodletting puncture. In traditional Chinese medicine, bloodletting puncture without cupping is often performed, especially in stroke patients in the acute stage or with impaired consciousness, and a recent clinical trial has recently been published showing that bloodletting puncture could provide clinical benefits to stroke patients with disturbance of consciousness ${ }^{38)}$. Further studies to compare and classify the effects of dry cupping, bloodletting puncture, and their combined form, wet cupping are required.

Generally, cupping therapy has been considered safe $^{33)}$, but AEs including anemia, infection, and scar formation have been reported ${ }^{39,40)}$. There have also been concerns that it might actually cause stroke ${ }^{41)}$. However, this study detected no serious AEs related to cupping therapy and demonstrated that the incidence of AEs in the additional cupping group was not significantly higher than that in the noncupping control group. This is consistent with the suggestions in previous studies that cupping therapy is a relatively safe intervention under the condition that it is carefully performed by experienced practitioners 40). However, considering that the meta-analysis on the occurrence of AEs in this study was based on only two small trials and that most of the reviewed studies did not consider the safety profile of cupping therapy in the process of study design, or omitted the reporting of AEs in the articles, we are hesitant to draw definitive conclusions about the safety of cupping therapy here. In particular, considering that there have been concerns ${ }^{41-43)}$ that cupping therapy performed on the neck area might cause arterial dissection by excessively raising the blood pressure of the carotid artery or vertebral artery that supplies cerebral circulation and eventually causes stroke, practitioners need to be careful not to induce excessive stress on the inside of the neck when performing cupping therapy in the neck area for stroke survivors.

This study has several limitations. First, despite our best efforts to secure as many relevant articles as possible, there may have been related trials that were not discovered by the search strategy in this study. We searched some databases based in Korea and China in addition to globally used core databases. This approach may have resulted in not identifying local publications in other regions, such as the Middle East or Africa, where cupping therapy has been used as a traditional intervention.

Second, there was a problem regarding the quantitative limitations of the included studies. Since there was no case in which the meta -analysis included more than 10 original trials, we could not construct a funnel plot to explore the existence of publication bias. Moreover, the scale of individual trials was too small. None of the trials had more than 100 participants per group. Crucially, no studies had previously calculated the optimal sample size for adequate power.

Third, the reporting quality of the included studies was low. Most studies did not clearly disclose how they corrected for the biases related to the random sequence generation, allocation concealment, dropout, and data selection. Most of 
them also omitted the reporting of AEs, as mentioned above. These omissions in reporting deteriorated the overall quality of the methodology of each trial and consequently lowered the certainty of the evidence derived from this systematic review and meta-analysis.

Fourth, none of the included studies considered blinding. Some researchers have attempted to develop a sham or minimal cupping device as a control intervention for cupping therapy ${ }^{44-46)}$. However, no sham/minimal cupping device has been confirmed to be perfectly suitable for blinding patients or practitioners ${ }^{44-46}$. Nevertheless, at least assessor blinding should have been ensured as a minimum measure to reduce detection bias. However, none of the included studies mentioned assessor blinding.

Fifth, the underlying mechanisms of the therapeutic effects of cupping therapy in the context of stroke has not been clearly determined. A previous systematic review ${ }^{6)}$ speculated that the benefit of cupping therapy for stroke rehabilitation may be due to the excretion of excess fluid and toxins and improvements in subcutaneous blood flow. To date, however, all of the hypotheses remain unclear.

Sixth, no conclusion was drawn about the influence of cupping therapy on critical outcomes directly related to stroke itself. In particular, as there are no long-term follow-up outcomes, the effects of cupping therapy on mortality, severe disability, and recurrence of stroke is still unexplored.

Finally, a large number of studies have adopted response rates as the main outcome measure instead of the internationally accepted standardized endpoints. This issue is one of the well-known chronic problems of RCTs dealing with cupping therapy ${ }^{33)}$. It can be a factor that prevents readers worldwide from trusting and adequately understanding the study results.

\section{Conclusion}

The findings of this study demonstrated the potential of cupping therapy to be beneficial in managing a variety of complications in stroke survivors. In particular, wet cupping therapy as an add-on for stroke survivors receiving conventional treatment provided significant benefits in improving upper limb motor function and spasticity, and these findings were supported by a moderate level of evidence. It was also found that cupping therapy did not significantly increase the occurrence of AEs, although the level of supporting evidence was very low. However, considering that the overall quality of the methodology of the included studies was low, further large-scale RCTs with rigorous designs are warranted to draw definite conclusions on the effectiveness and safety of cupping therapy for stroke survivors.

\section{Conflicts of interest}

The authors declare that they have no competing interests.

\section{Acknowledgments}

This study was undertaken as part of the KHIDI, funded by the Ministry of Health \& Welfare, Republic of Korea. The funders did not 
play any role in the design and conduct of this study.

\section{Author contributions}

Conceptualization: $\mathrm{CH}$. Methodology: $\mathrm{MK}$ and $\mathrm{CH}$. Formal Analysis: $\mathrm{MK}$ and $\mathrm{CH}$. Wiring Original Draft: MK. Writing - Reviewing \& Editing: $\mathrm{MK}$ and $\mathrm{CH}$. Supervision: $\mathrm{CH}$. Project Acquisition: $\mathrm{CH}$

\section{Funding}

This research was supported by a grant from the Korean Technology R\&D Project through the Korea Health Industry Development Institute (KHIDI), funded by the Ministry of Health \& Welfare, Republic of Korea (HB16C00001).

\section{Ethical statement}

This study did not involve any human or animal experiment.

\section{Data availability}

The data that support the findings of this study are available from the corresponding author upon reasonable request.

\section{References}

1. Naghavi, M., Abajobir, A. A., Abbafati, C., Abbas, K. M., Abd-Allah, F., \& Abera, S. F., et al. (2017). Global, regional, and national age-sex specific mortality for 264 causes of death, 1980-2016: a systematic analysis for the Global Burden of Disease Study 2016. Lancet, 390(10100), 1151-1210. doi: 10.1016/ S0140-6736(17)32152-9.

2. Gresham, G. E., Fitzpatrick, T. E., Wolf, P. A., McNamara, P. M., Kannel, W. B., \& Dawber, T. R. (1975). Residual Disability in Survivors of Stroke - The Framingham Study. New England Journal of Medicine, 293(19), 954-956. doi: 10.1056/NEJM197511 062931903.

3. Ovbiagele, B., Goldstein, L. B., Higashida, R. T., Howard, V. J., Johnston, S. C., \& Khavjou, O. A., et al. (2013). Forecasting the future of stroke in the United States: a policy statement from the American Heart Association and American Stroke Association. Stroke, 44(8), 2361-2375. doi: 10.1161/STR. 0b013e31829734f2.

4. Shah, S. H., Engelhardt, R., \& Ovbiagele, B. (2008). Patterns of complementary and alternative medicine use among United States stroke survivors. Journal of the Neurological Sciences, 271(1), 180-185. doi: 10.1016/j.jns. 2008.04.014.

5. Al-Bedah, A. M., Aboushanab, T. S., Alqaed, M. S., Qureshi, N. A., Suhaibani, I., \& Ibrahim, G., et al. (2016). Classification of cupping therapy: a tool for modernization and standardization. Journal of Complementary and Alternative Medical Research, 1(1), 1-10. doi: 10.9734/JOCAMR/2016/27222.

6. Lee, M. S., Choi, T. Y., Shin, B. C., Han, C., \& Ernst, E. (2010). Cupping for stroke rehabilitation: a systematic review. Journal of the Neurological Sciences, 294(1-2), 70-73. 
doi: 10.1016/j.jns.2010.03.033.

7. Higgins, J. P., \& Thompson, S. G. (2002). Quantifying heterogeneity in a meta-analysis. Statistics in Medicine, 21(11),1539-1558. doi: 10.1002/sim.1186.

8. Higgins, J. P., Thomas, J., Chandler, J., Cumpston, M., Li, T., \& Page, M. J., et al. (2019). Cochrane handbook for systematic reviews of interventions (2nd ed.). John Wiley \& Sons.

9. Sterne, J. A., Savović, J., Page, M. J., Elbers, R. G., Blencowe, N.S., \& Boutron, I., et al. (2019). RoB 2: a revised tool for assessing risk of bias in randomised trials. BMJ, 366. doi: 10.1136/bmj.14898.

10. Guyatt, G. H., Oxman, A. D., Schünemann, H. J., Tugwell, P., \& Knottnerus, A. (2011). GRADE guidelines: A new series of articles in the Journal of Clinical Epidemiology. Journal of Clinicial Epidemiology, 64(4), 380-382. doi: 10.1016/j.jclinepi.2010.09.011.

11. Ji, P. B., Wu, Z. Q., Jia, D. P., Wang, L., \& Shu, Y. Y. (2020). Clinical Study of Colored Silica Gel Can Combined with Exercise Therapy for Unilateral Neglect After Stroke. Shanghai Journal of Acupuncture and Moxibustion, 39(8), 983-987. doi: 10.13460/ j.issn.1005-0957.2020.08.0983

12. Zhang, P., Chen, Y., Dai, W., \& Nie, L. (2020). Observation on the effect of puncturing and cupping combined with rehabilitation training in the treatment of stroke patients with upper limb elbow flexion spasm and analysis of the effect of biceps integrated electromyography. China Rural Health, 12(20), 75.

13. Li, Y. (2019). Clinical Study of Blood - letting Puncture and Cupping Combined with Bobath Technique in the Treatment of Upper Limb Spasticity after Stroke. Chinese Journal of Ethnomedicine and Ethnopharmacy, 28(21), 84-86.

14. Shan, Y., Liu, J., Sun, L., Li, Q., \& Wang, J. (2019). Therapeutic Observation of Collateral -pricking Cupping for Upper-limb Spasm in Patients with Cerebral Stroke. Shanghai Journal of Acupuncture and Moxibustion, 38(3), 270-274. doi: 10.13460/j.issn.1005-0957. 2019.03.0270.

15. Jiang, L. (2018). The Curative Effect of Acupuncture and Cupping Therapy on Upper Limb Dysfunction of Stroke Xinjiang Medical University [Master's thesis].

16. Huang, Z., Zhao, N., Su, Z., Su, J., \& Wu, Q. (2018). Effects of pricking and cupping combined with rehabilitation training on elbow flexion spasticity of upper limb after stroke and its IEMG value. Chinese Acupuncture \& Moxibustion, 38(2), 119-124. doi: $10.13703 /$ j.0255-2930.2018.02.002.

17. Chen, J., Lin, S. (2017). The clinical effect of pricking and cupping combined with rehabilitation training in the treatment of stroke patients with upper limb elbow flexion spasm. Chinese Journal of Medical Device, 30(15), 121-122.

18. Chen, Y. P. (2017). Clinical Research on Hemidysesthesia After Stroke Treated by Acupuncture combined with Collateral-Needling Cupping. Henan Traditional Chinese Medicine, 37(1), 87-89. doi: 10.16367/j.issn.1003-5028. 2017.01.0029.

19. Liu, H., Liu, T., \& Wang, Z. (2016). Effect 
of acupoint injection combined with cupping treatment on the daily life ability of patients with shoulder pain after stroke. Chinese Community Doctors, 32(27), 169-170. doi: 10.3969/j.issn.1007-614x.2016.27.108.

20. Zhang, B. (2016). The Clinical Observation of Body Spasm After Stroke Applying The Therapy of Combination Acupucture With Pricking and Cupping. Shandong University of Traditional Chinese Medicine [Master's thesis].

21. Cheng, X., Cheng, C. (2014). Cupping combined Conventional Symptomatic Treatment and Rehabilitation Training after Stroke Shoulder - hand Syndrome Randomized Parallel Group Study. Journal of Practical Traditional Chinese Internal Medicine, 28(11), 27-29. doi: 10.13729/j.issn.1671-7813.2014.11.12.

22. Zhang, Q., Wei, Z., Cui, Y. (2014), Bloodletting and cupping combined with exercise therapy to treat 31 cases of stroke and hemiplegia and shoulder pain. Hebei Journal of Traditional Chinese Medicine, 36(4), 568-569.

23. Miao, T. (2014). MOTOmed intelligent exercise system combined with blood pricking and cupping for treatment of increased muscle tone of lower limbs in stroke patients. Practical Journal of Medicine and Pharmacy, 31(3), 214-215. doi: 10.14172/j.cnki.issn1671 $-4008.2014 .03 .007$.

24. Huang, Z. (2014). Effect of Pricking Blood with Cupping Therapy Combined with Rehabilitation Training on the Spasticity of the Upper Limb in Patients with Stroke. Fujian University of Traditional Chinese Medicine [Master's thesis].
25. Yang, M. (2013). Rehabilitation training combined with cupping to treat 64 cases of shoulder pain after stroke. Forum on Traditional Chinese Medicine, 28(6), 26-27.

26. Gao, L., Chu, J., \& Bao, Y. (2012). Observation on the effect of blood puncturing and cupping combined with rehabilitation on hemiplegic shoulder pain and upper limb motor function improvement after stroke. Zhejiang Journal of Traditional Chinese Medicine, 47(11), 821-823.

27. Peng, L., Wang, Z., Li, L. (2012). Research of Shoulder Hand Syndrome After Stroke Treated by Collateral Disease Theory. Journal of Emergency in Traditional Chinese Medicine, 21(3), 448-449.

28. Wu, Z., \& Huang, W. (2011). Observation of puncture and cupping combined with rehabilitation training in the treatment of apoplexy and shoulder pain. Journal of Zhejiang Chinese Medical University, 35(3), 425. doi: 10.16466/j.issn1005-5509.2011.03.013.

29. Fu, L., Liu, W., Wu, Q., Li, X., Li, D., \& Shi, X., et al. (2009). Observations on the Efficacy of Acupuncture Plus Pricking-cupping Bloodletting in Treating Postapoplectic Shoulder-hand Syndrome. Shanghai Journal of Acupuncture and moxibustion, 28(3), 132-134. doi: 10.13460/j.issn.1005-0957.2009. 03.003.

30. Zou, C., Zhu, G., \& Bao, Y. (2006). Treatment of 30 Cases of Shoulder-Hand Syndrome after Apoplexy with Acupuncture and Collateral Puncture Cupping. Zhejiang Journal of Traditional Chinese Medicine, 41(6), 340. 
31. Ding, B., \& Cui, Y. (2003). Treatment of Hemiplegia and Joint Contracture after Apoplexy by Acupuncture plus Cupping Therapy: A Report of 52 Cases. Journal of Acupucture and Tuina Science, 1(5), 38-39. doi: 10.1007/BF02874843.

32. Cui, S. (2001). Observation on the Curative Effect of Plum-blossom Needle and Cupping in Treating Sequelae. Journal of External Therapy of Traditional Chinese Medicine, 10(4), 37.

33. Cao, H., Li, X., \& Liu, J. (2012). An updated review of the efficacy of cupping therapy. PLoS One, 7(2), e31793. doi: 10.1371/journal. pone. 0031793 .

34. Lee. M. S., Kim, J. I., \& Ernst, E. (2011). Is cupping an effective treatment? An overview of systematic reviews. Journal of Acupuncture and Meridian Studies, 4(1), 1-4. doi: 10.1016/ S2005-2901(11)60001-0.

35. Guo, Y., Chen, B., Wang, D., Li, M., Lim, C. H., \& Guo Y, et al. (2017). Cupping regulates local immunomodulation to activate neural -endocrine-immune worknet. Complementary Therapies in Clinical Practice, 28, 1-3. doi: 10.1016/j.ctcp.2017.04.005.

36. Lowe, D. T. (2017). Cupping therapy: An analysis of the effects of suction on skin and the possible influence on human health. Complementary Therapies in Clinical Practice, 29, 162-168. doi: 10.1016/j.ctcp.2017.09.008.

37. Bridgett, R., Klose, P., Duffield, R., Mydock, S., \& Lauche, R. (2018). Effects of Cupping Therapy in Amateur and Professional Athletes: Systematic Review of Randomized Controlled Trials. Journal of Alternative and Complementary
Medicine, 24(3), 208-219. doi: 10.1089/acm. 2017.0191

38. Yu, N. N., Xu, Z. F., Gao, Y., Zhou, Z.L., Zhao, X., \& Zhou, D., et al. (2021). Wake-Promoting Effect of Bloodletting Puncture at Hand Twelve Jing-Well Points in Acute Stroke Patients: A Multi-center Randomized Controlled Trial. Chinese Journal of Integrative Medicine, 27(8), 570-577. doi: 10.1007/s11655-020-3093-8.

39. Wang, S. Z., Lu, Y. H., Wu, M., Chen, K. J., Liu, Y., \& Liu, L. T. (2021). Cupping Therapy for Diseases: An Overview of Scientific Evidence from 2009 to 2019. Chinese Journal of Integrative Medicine, 27(5), 394-400. doi: 10.1007/s11655-020-3060-y.

40. Kim, T. H., Kim, K. H., Choi, J. Y., \& Lee, M.S. (2014). Adverse events related to cupping therapy in studies conducted in Korea: A systematic review. European Journal of Integrative Medicine, 6(4), 434-440. doi: 10.1016/j.eujim.2013.06.006.

41. Blunt, S. B., \& Lee, H. P. (2010). Can traditional "cupping" treatment cause a stroke?. Medical Hypotheses, 74(5), 945-949. doi: 10.1016/j.mehy.2009.11.037.

42. Choi, J. Y., Huh, C. W., Choi, C.H., \& Lee, J. I. (2016). Extracranial vertebral artery rupture likely secondary to "cupping therapy" superimposed on spontaneous dissection. Interventional Neuroradiology, 22(6), 728-731. doi: $10.1177 / 1591019916659264$.

43. Zuhorn, F., Schäbitz, W. R., Oelschläger, C., Klingebiel, R., \& Rogalewski, A. (2020). Cervical Artery Dissection Caused by Electrical Cupping Therapy with High-Negative Pressure - Case Report. Journal of Stroke and 
Cerebrovascular Diseases, 29(11), 105207. doi: $10.1016 /$ j.jstrokecerebrovasdis.2020.105207.

44. Lauche, R., Spitzer, J., Schwahn, B., Ostermann, T., Bernardy, K., \& Cramer, H., et al. (2016). Efficacy of cupping therapy in patients with the fibromyalgia syndrome-a randomised placebo controlled trial. Scientific Report, 6, 37316. doi: 10.1038/srep37316.

45. Lee, M. S., Kim, J. I., Kong, J. C., Lee, D. H., \& Shin, B. C. (2010). Developing and validating a sham cupping device. Acupuncture in Medicine, 28(4), 200-204. doi: 10.1136/aim. 2010.002329.

46. Teut, M., Ullmann, A., Ortiz, M., Rotter, G.,
Binting, S., \& Cree, M., et al. (2018). Pulsatile dry cupping in chronic low back pain - a randomized three-armed controlled clinical trial. BMC Complementary and Alternative Medicine, 18(1), 115. doi: 10.1186/s12906-018-2187-8.

\section{ORCID}

Mikyung Kim https://orcid.org/0000-0002-9912-6249

Chang-ho Han https://orcid.org/0000-0003-3460-9263 
Supplement 1. Electronic database and search term

1. PubMed (https://www.ncbi.nlm.nih.gov/)

$((((((($ stroke[Title/Abstract]) OR (cerebral infarction[Title/Abstract])) OR (cerebral hemorrhage[Title/Abstract])) OR (cerebrovascular disorder*[Title/Abstract])) OR (cerebrovascular accident*[Title/Abstract])) OR (apoplexy[Title/Abstract])) OR (brain ischemia[Title/Abstract]) ) OR (((((cerebral infarction[MeSH Terms]) OR (cerebral hemorrhage[MeSH Terms])) OR (cerebrovascular accident[MeSH Terms])) OR (cerebrovascular disorder[MeSH Terms])) OR (apoplexy, cerebrovascular[MeSH Terms])) OR (apoplexy[MeSH Terms])) AND ((cupping [MeSH Terms]) OR (cupping [Title/Abstract]))

2. Embase (https://www.embase.com/)

(stroke:ti,ab,kw OR 'cerebral infarction':ti,ab,kw OR 'cerebral hemorrhage'ti,ab,kw OR 'cerebrovascular disorder':ti,ab,kw OR 'cerebrovascular disease':ti,ab,kw OR 'cerebrovascular accident':ti,ab,kw OR apoplexy:ti,ab,kw OR 'cerebrovascular accident'/exp OR 'stroke patient'/exp OR 'brain infarction'/exp OR 'brain hemorrhage'/exp OR 'cerebrovascular disease'/exp) AND (cupping:ti,ab,kw OR 'cupping therapy'/exp)

3. Cochrane Library (https://www.cochranelibrary.com/)

[TiAbKw] (stroke OR 'cerebral infarction' OR 'cerebral hemorrhage' OR 'cerebrovascular disorder' OR 'cerebrovascular accident' OR apoplexy OR 'brain ischemia') AND (cupping)

4. China Academic Journals (https://oversea.cnki.net/index/)

[TiKwAb] [Precise] (中风 OR 卒中 OR 脑卒中 $\mathrm{OR}$ 脑梗死 OR 脑栓塞 OR 脑溢血 OR 脑出血 OR 缺血性脑卒中 $\mathrm{OR}$ 出血性脑卒中 OR 脑血管) AND (拔罐)

5. Science On (https://scienceon.kisti.re.kr/)

6. Korean Studies Information Service System (http://kiss.kstudy.com/)

7. OASIS (https://oasis.kiom.re.kr/)

[TiAbKw] (뇌졸중 OR 뇌경색 OR 뇌출혈 OR 중풍 OR 뇌혈관질환) AND (부항) 
Supplement 2. PRISMA 2020 abstract checklist

\begin{tabular}{|c|c|c|c|}
\hline Section and Topic & Item \# & Checklist item & $\begin{array}{l}\text { Reported } \\
(\text { Yes/No) }\end{array}$ \\
\hline \multicolumn{4}{|c|}{ TITLE } \\
\hline Title & 1 & Identify the report as a systematic review. & Y \\
\hline \multicolumn{4}{|l|}{ BACKGROUND } \\
\hline Objectives & 2 & $\begin{array}{l}\text { Provide an explicit statement of the main objective(s) or question(s) the review } \\
\text { addresses. }\end{array}$ & Y \\
\hline \multicolumn{4}{|l|}{ METHODS } \\
\hline Eligibility criteria & 3 & Specify the inclusion and exclusion criteria for the review. & $\mathrm{Y}$ \\
\hline Information sources & 4 & $\begin{array}{l}\text { Specify the information sources (e.g. databases, registers) used to identify studies and } \\
\text { the date when each was last searched. }\end{array}$ & Y \\
\hline Risk of bias & 5 & Specify the methods used to assess risk of bias in the included studies. & Y \\
\hline Synthesis of results & 6 & Specify the methods used to present and synthesise results. & Y \\
\hline \multicolumn{4}{|l|}{ RESULTS } \\
\hline Included studies & 7 & $\begin{array}{l}\text { Give the total number of included studies and participants and summarise relevant } \\
\text { characteristics of studies. }\end{array}$ & Y \\
\hline Synthesis of results & 8 & $\begin{array}{l}\text { Present results for main outcomes, preferably indicating the number of included } \\
\text { studies and participants for each. If meta-analysis was done, report the summary } \\
\text { estimate and confidence/credible interval. If comparing groups, indicate the direction } \\
\text { of the effect (i.e. which group is favoured). }\end{array}$ & Y \\
\hline \multicolumn{4}{|l|}{ DISCUSSION } \\
\hline $\begin{array}{l}\text { Limitations of } \\
\text { evidence }\end{array}$ & 9 & $\begin{array}{l}\text { Provide a brief summary of the limitations of the evidence included in the review } \\
\text { (e.g. study risk of bias, inconsistency and imprecision). }\end{array}$ & $\begin{array}{c}\mathrm{Y} \\
\text { (conclusion) }\end{array}$ \\
\hline Interpretation & 10 & Provide a general interpretation of the results and important implications. & $\begin{array}{c}\mathrm{Y} \\
\text { (conclusion) }\end{array}$ \\
\hline \multicolumn{4}{|l|}{ OTHER } \\
\hline Funding & 11 & Specify the primary source of funding for the review. & NA \\
\hline Registration & 12 & Provide the register name and registration number. & NA \\
\hline
\end{tabular}

From: Page MJ, McKenzie JE, Bossuyt PM, Boutron I, Hoffmann TC, Mulrow CD, et al. The PRISMA 2020 statement: an updated guideline for reporting systematic reviews. BMJ 2021;372:n71. doi: 10.1136/bmj.n71 
Supplement 3. PRISMA 2020 checklist

\begin{tabular}{|c|c|c|c|}
\hline Section and Topic & Item \# & Checklist item & $\begin{array}{l}\text { Location where item is } \\
\text { reported }\end{array}$ \\
\hline \multicolumn{4}{|c|}{ TITLE } \\
\hline Title & 1 & Identify the report as a systematic review. & P1. title \\
\hline \multicolumn{4}{|l|}{ ABSTRACT } \\
\hline Abstract & 2 & See the PRISMA 2020 for Abstracts checklist. & P2 \& Supple 2 \\
\hline \multicolumn{4}{|l|}{ INTRODUCTION } \\
\hline Rationale & 3 & $\begin{array}{l}\text { Describe the rationale for the review in the context of existing } \\
\text { knowledge. }\end{array}$ & P3, line 2-19 \\
\hline Objectives & 4 & $\begin{array}{l}\text { Provide an explicit statement of the objective(s) or question(s) the } \\
\text { review addresses. }\end{array}$ & P3, line 20-22 \\
\hline \multicolumn{4}{|l|}{ METHODS } \\
\hline Eligibility criteria & 5 & $\begin{array}{l}\text { Specify the inclusion and exclusion criteria for the review and how } \\
\text { studies were grouped for the syntheses. }\end{array}$ & P4. Chapter 2.2 \\
\hline Information sources & 6 & $\begin{array}{l}\text { Specify all databases, registers, websites, organisations, reference } \\
\text { lists and other sources searched or consulted to identify studies. } \\
\text { Specify the date when each source was last searched or consulted. }\end{array}$ & P4. Chapter2.1 \\
\hline Search strategy & 7 & $\begin{array}{l}\text { Present the full search strategies for all databases, registers and } \\
\text { websites, including any filters and limits used. }\end{array}$ & $\begin{array}{l}\text { Supple } 3 \& \text { P4. Chapter } \\
2.1 .2\end{array}$ \\
\hline Selection process & 8 & $\begin{array}{l}\text { Specify the methods used to decide whether a study met the inclusion } \\
\text { criteria of the review, including how many reviewers screened each } \\
\text { record and each report retrieved, whether they worked independently, } \\
\text { and if applicable, details of automation tools used in the process. }\end{array}$ & P4. Chapter 2.2.1 \\
\hline Data collection process & 9 & $\begin{array}{l}\text { Specify the methods used to collect data from reports, including } \\
\text { how many reviewers collected data from each report, whether they } \\
\text { worked independently, any processes for obtaining or confirming } \\
\text { data from study investigators, and if applicable, details of automation } \\
\text { tools used in the process. }\end{array}$ & P5. Chapter 2.3. \\
\hline \multirow[t]{2}{*}{ Data items } & $10 \mathrm{a}$ & $\begin{array}{l}\text { List and define all outcomes for which data were sought. Specify } \\
\text { whether all results that were compatible with each outcome domain } \\
\text { in each study were sought (e.g. for all measures, time points, analyses), } \\
\text { and if not, the methods used to decide which results to collect. }\end{array}$ & P5. Chapter 2.2.6 \\
\hline & $10 \mathrm{~b}$ & $\begin{array}{l}\text { List and define all other variables for which data were sought (e.g. } \\
\text { participant and intervention characteristics, funding sources). Describe } \\
\text { any assumptions made about any missing or unclear information. }\end{array}$ & P5, line 2-4 (Chapter 2.3.) \\
\hline $\begin{array}{l}\text { Study risk of bias } \\
\text { assessment }\end{array}$ & 11 & $\begin{array}{l}\text { Specify the methods used to assess risk of bias in the included } \\
\text { studies, including details of the tool(s) used, how many reviewers } \\
\text { assessed each study and whether they worked independently, and if } \\
\text { applicable, details of automation tools used in the process. }\end{array}$ & P5. Chapter 2.5 \\
\hline Effect measures & 12 & $\begin{array}{l}\text { Specify for each outcome the effect measure(s) (e.g. risk ratio, } \\
\text { mean difference) used in the synthesis or presentation of results. }\end{array}$ & P5. Chapter 2.4 \\
\hline \multirow[t]{2}{*}{ Synthesis methods } & $13 \mathrm{a}$ & $\begin{array}{l}\text { Describe the processes used to decide which studies were eligible } \\
\text { for each synthesis (e.g. tabulating the study intervention } \\
\text { characteristics and comparing against the planned groups for each } \\
\text { synthesis (item \#5)). }\end{array}$ & P5. Chapter 2.4 \\
\hline & $13 b$ & $\begin{array}{l}\text { Describe any methods required to prepare the data for presentation } \\
\text { or synthesis, such as handling of missing summary statistics, or data } \\
\text { conversions. }\end{array}$ & $\begin{array}{l}\text { Not done (The data } \\
\text { presented in a form that } \\
\text { could be quantitatively } \\
\text { synthesized were chosen) }\end{array}$ \\
\hline
\end{tabular}




\begin{tabular}{|c|c|c|c|}
\hline Section and Topic & Item \# & Checklist item & $\begin{array}{l}\text { Location where item is } \\
\text { reported }\end{array}$ \\
\hline & $13 \mathrm{c}$ & $\begin{array}{l}\text { Describe any methods used to tabulate or visually display results of } \\
\text { individual studies and syntheses. }\end{array}$ & $\begin{array}{l}\text { Not done (The results were } \\
\text { presented in the most } \\
\text { widely used methods; } \\
\text { Tables for individual } \\
\text { studies and forest plots for } \\
\text { synthesized data) }\end{array}$ \\
\hline & $13 \mathrm{~d}$ & $\begin{array}{l}\text { Describe any methods used to synthesize results and provide a } \\
\text { rationale for the choice(s). If meta-analysis was performed, describe } \\
\text { the model(s), method(s) to identify the presence and extent of } \\
\text { statistical heterogeneity, and software package(s) used. }\end{array}$ & P5. Chapter 2.4 \\
\hline & $13 \mathrm{e}$ & $\begin{array}{l}\text { Describe any methods used to explore possible causes of heterogeneity } \\
\text { among study results (e.g. subgroup analysis, meta-regression). }\end{array}$ & P5. Chapter 2.4 \\
\hline & $13 \mathrm{f}$ & $\begin{array}{l}\text { Describe any sensitivity analyses conducted to assess robustness of } \\
\text { the synthesized results. }\end{array}$ & Not done \\
\hline $\begin{array}{l}\text { Reporting bias } \\
\text { assessment }\end{array}$ & 14 & $\begin{array}{l}\text { Describe any methods used to assess risk of bias due to missing } \\
\text { results in a synthesis (arising from reporting biases). }\end{array}$ & $\begin{array}{l}\text { P5. Chapter 2.4Followed the } \\
\text { methods of RoB 2. Its third } \\
\text { domain is about the missing } \\
\text { outcome data.(Fig 2) }\end{array}$ \\
\hline Certainty assessment & 15 & $\begin{array}{l}\text { Describe any methods used to assess certainty (or confidence) in the } \\
\text { body of evidence for an outcome. }\end{array}$ & P6. Chapter 2.6 \\
\hline \multicolumn{4}{|l|}{ RESULTS } \\
\hline \multirow[t]{2}{*}{ Study selection } & $16 \mathrm{a}$ & $\begin{array}{l}\text { Describe the results of the search and selection process, from the } \\
\text { number of records identified in the search to the number of studies } \\
\text { included in the review, ideally using a flow diagram. }\end{array}$ & P7. Chapter 3.1 \& Fig. 1 \\
\hline & $16 \mathrm{~b}$ & $\begin{array}{l}\text { Cite studies that might appear to meet the inclusion criteria, but } \\
\text { which were excluded, and explain why they were excluded. }\end{array}$ & $\begin{array}{l}\text { P7. Chapter } 3.1 \& \text { Fig. } 1 \& \\
\text { Supple } 4\end{array}$ \\
\hline Study characteristics & 17 & Cite each included study and present its characteristics. & $\begin{array}{l}\text { P7. Chapter 3.1., reference } \\
\text { no. 11-32 }\end{array}$ \\
\hline Risk of bias in studies & 18 & Present assessments of risk of bias for each included study. & P8. Chapter $3.3 \&$ Fig $2 \& 3$ \\
\hline $\begin{array}{l}\text { Results of individual } \\
\text { studies }\end{array}$ & 19 & $\begin{array}{l}\text { For all outcomes, present, for each study: (a) summary statistics for } \\
\text { each group (where appropriate) and (b) an effect estimate and its } \\
\text { precision (e.g. confidence/credible interval), ideally using structured } \\
\text { tables or plots. }\end{array}$ & Table 2, Fig.4-12 \\
\hline \multirow[t]{4}{*}{ Results of syntheses } & $20 \mathrm{a}$ & $\begin{array}{l}\text { For each synthesis, briefly summarise the characteristics and risk of } \\
\text { bias among contributing studies. }\end{array}$ & $\begin{array}{l}\text { P9. Chapter } 3.4 \& \text { Supple } \\
5 \& 6\end{array}$ \\
\hline & $20 \mathrm{~b}$ & $\begin{array}{l}\text { Present results of all statistical syntheses conducted. If meta-analysis } \\
\text { was done, present for each the summary estimate and its precision } \\
\text { (e.g. confidence/credible interval) and measures of statistical } \\
\text { heterogeneity. If comparing groups, describe the direction of the } \\
\text { effect. }\end{array}$ & $\begin{array}{l}\text { P9. Chapter } 3.4 \text { \& Fig. } 4-12 \\
\text { \& Supple } 5 \& 6\end{array}$ \\
\hline & $20 \mathrm{c}$ & $\begin{array}{l}\text { Present results of all investigations of possible causes of } \\
\text { heterogeneity among study results. }\end{array}$ & $\begin{array}{l}\text { P9. subgroup analysis in } \\
\text { Chapter 3.4) \& Fig.4-12 }\end{array}$ \\
\hline & $20 \mathrm{~d}$ & $\begin{array}{l}\text { Present results of all sensitivity analyses conducted to assess the } \\
\text { robustness of the synthesized results. }\end{array}$ & Not done \\
\hline Reporting biases & 21 & $\begin{array}{l}\text { Present assessments of risk of bias due to missing results (arising } \\
\text { from reporting biases) for each synthesis assessed. }\end{array}$ & $\begin{array}{l}\text { Fig } 2 \text { - RoB2, Domain } 3 \text {. } \\
\text { missing outcome data }\end{array}$ \\
\hline Certainty of evidence & 22 & $\begin{array}{l}\text { Present assessments of certainty (or confidence) in the body of } \\
\text { evidence for each outcome assessed. }\end{array}$ & $\begin{array}{l}\text { P9. Chapter 3.4) \& Supple } \\
5 \& 6\end{array}$ \\
\hline
\end{tabular}




\begin{tabular}{|c|c|c|c|}
\hline Section and Topic & Item \# & Checklist item & $\begin{array}{l}\text { Location where item is } \\
\text { reported }\end{array}$ \\
\hline \multicolumn{4}{|l|}{ DISCUSSION } \\
\hline \multirow[t]{4}{*}{ Discussion } & $23 \mathrm{a}$ & $\begin{array}{l}\text { Provide a general interpretation of the results in the context of other } \\
\text { evidence. }\end{array}$ & $\begin{array}{l}\text { P12. 3rd paragraph-P13. } \\
\text { 4th paragraph }\end{array}$ \\
\hline & $23 b$ & Discuss any limitations of the evidence included in the review. & P14 \\
\hline & $23 \mathrm{c}$ & Discuss any limitations of the review processes used. & P13. last paragraph \\
\hline & $23 \mathrm{~d}$ & $\begin{array}{l}\text { Discuss implications of the results for practice, policy, and future } \\
\text { research. }\end{array}$ & P15. Chapter 5 \\
\hline \multicolumn{4}{|l|}{ OTHER INFORMATION } \\
\hline \multirow[t]{3}{*}{ Registration and protocol } & $24 \mathrm{a}$ & $\begin{array}{l}\text { Provide registration information for the review, including register } \\
\text { name and registration number, or state that the review was not } \\
\text { registered. }\end{array}$ & P14 line $15-16$ \\
\hline & $24 \mathrm{~b}$ & $\begin{array}{l}\text { Indicate where the review protocol can be accessed, or state that a } \\
\text { protocol was not prepared. }\end{array}$ & P14 line 15-16 \\
\hline & $24 \mathrm{c}$ & $\begin{array}{l}\text { Describe and explain any amendments to information provided at } \\
\text { registration or in the protocol. }\end{array}$ & P14 line $15-16$ \\
\hline Support & 25 & $\begin{array}{l}\text { Describe sources of financial or non-financial support for the } \\
\text { review, and the role of the funders or sponsors in the review. }\end{array}$ & P14 line $7-10$ \\
\hline Competing interests & 26 & Declare any competing interests of review authors. & P14 line 12-13 \\
\hline $\begin{array}{l}\text { Availability of data, code } \\
\text { and other materials }\end{array}$ & 27 & $\begin{array}{l}\text { Report which of the following are publicly available and where they } \\
\text { can be found: template data collection forms; data extracted from } \\
\text { included studies; data used for all analyses; analytic code; any other } \\
\text { materials used in the review. }\end{array}$ & P14 line $18-21$ \\
\hline
\end{tabular}




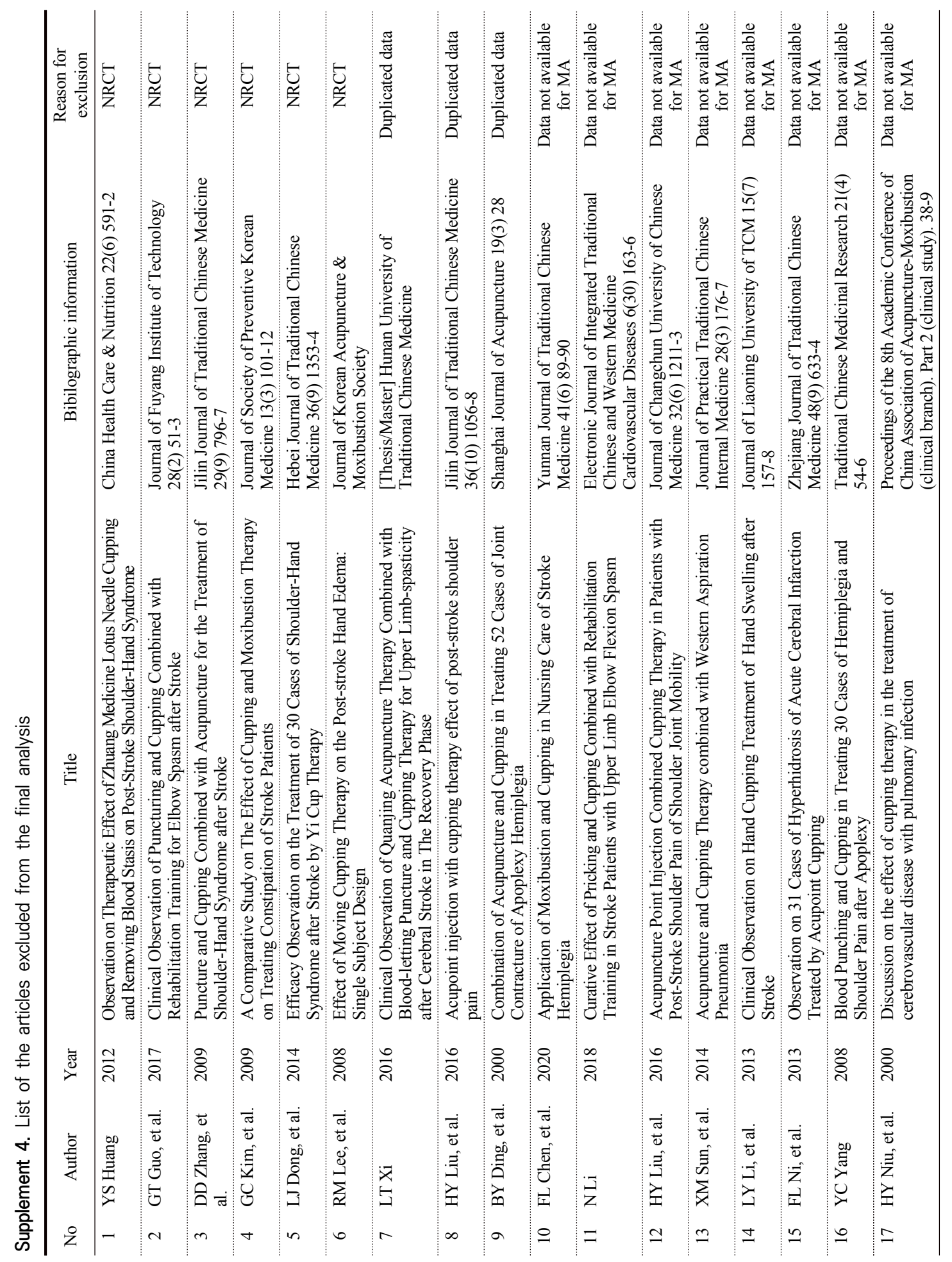




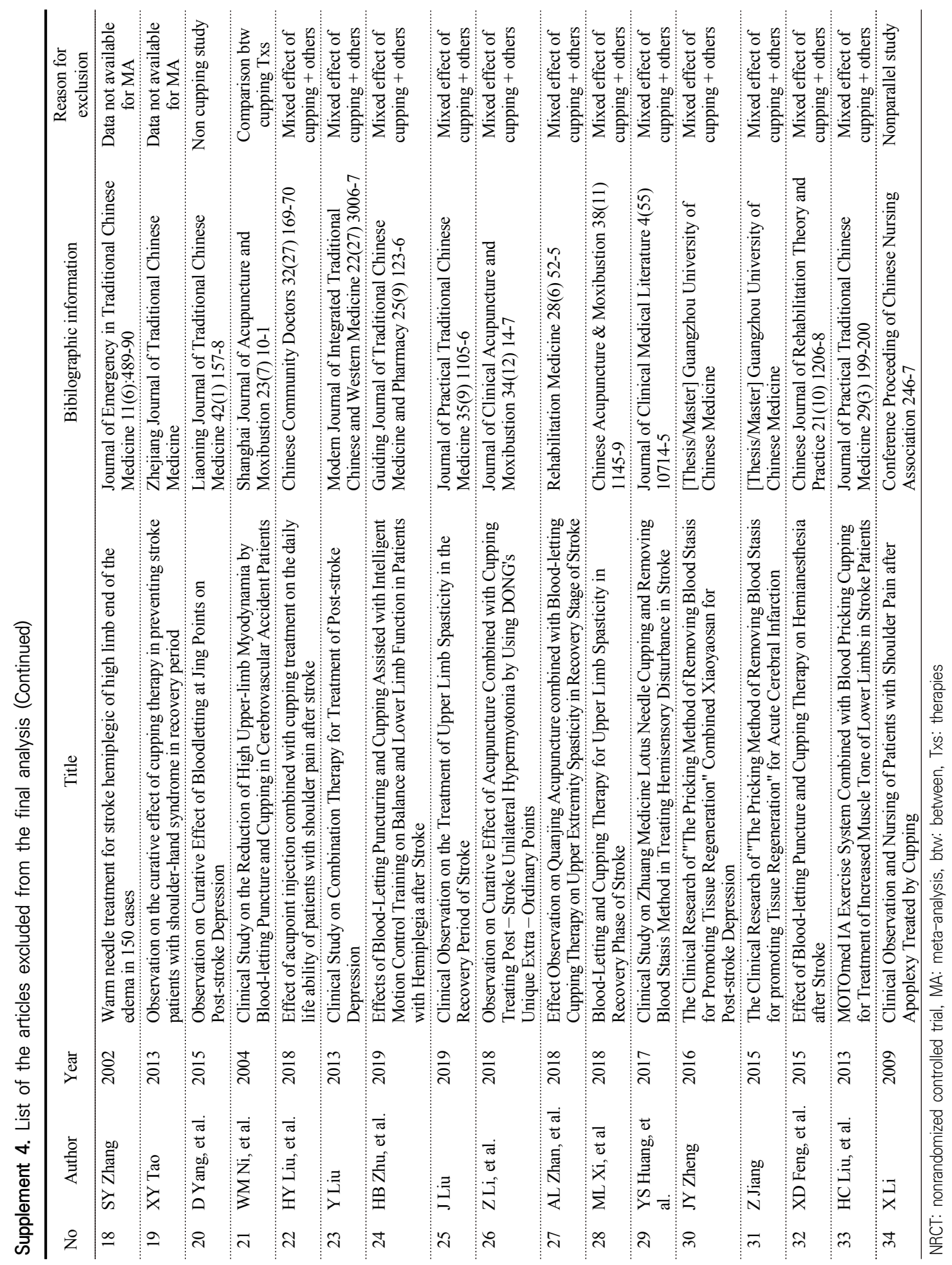


Supplement 5. Risk of bias summary of individual studies

\begin{tabular}{|c|c|c|c|c|c|c|}
\hline Study ID & D1 & $\underline{\mathrm{D} 2}$ & $\underline{\mathrm{D} 3}$ & $\underline{\mathrm{D} 4}$ & $\underline{\mathrm{D} 5}$ & Overall \\
\hline JiPB2020 & $!$ & + & & & $!$ & \\
\hline ZhangP2020 & $!$ & + & & & $!$ & \\
\hline LiYY2019 & $!$ & + & & & $!$ & \\
\hline ShanYL2019 & $!$ & + & & & $!$ & \\
\hline JiangL2018 & + & + & & & $!$ & \\
\hline HuangZQ2018 & ! & + & & & $!$ & \\
\hline ChenJY2017 & $!$ & + & & & $!$ & \\
\hline ChenYP2017 & $!$ & + & & & $!$ & \\
\hline LiuHY2016 & ! & + & & & $!$ & \\
\hline ZhangBB2016 & $!$ & + & & & $!$ & \\
\hline ChengXL2014 & $!$ & + & & & $!$ & \\
\hline ZhangQF2014 & ! & + & & & $!$ & \\
\hline MiaoT2014 & ! & + & & & $!$ & \\
\hline HuangZQ2014 & + & + & & & $!$ & \\
\hline YangMY2013 & ! & + & & & $!$ & \\
\hline GaoLA2015 & ! & + & & & $!$ & \\
\hline PengL2012 & $!$ & + & & & $!$ & \\
\hline WuZY2011 & $!$ & + & & & $!$ & \\
\hline FuL2009 & $!$ & + & & & $!$ & \\
\hline ZouC2006 & $!$ & + & & & $!$ & \\
\hline DingBY2003 & ! & + & & & ! & \\
\hline Cuiszzo01 & $!$ & + & & & ! & \\
\hline
\end{tabular}

\begin{tabular}{|c|l|}
\hline+ & Low risk \\
\hline$!$ & Some concerns \\
\hline & High risk \\
\hline D1 & Randomisation process \\
\hline D2 & Deviations from the intended interventions \\
\hline D3 & Missing outcome data \\
\hline D4 & Measurement of the outcome \\
\hline D5 & Selection of the reported result \\
\hline
\end{tabular}

Supplement 6. Overall risk of bias of included studies

\section{As percentage}

\section{Overall Bias}

Selection of the reported result

Measurement of the outcome

Mising outcome data

Deviations from intended interventions

Randomization process

- Low risk Some concerns $\square$ High risk 


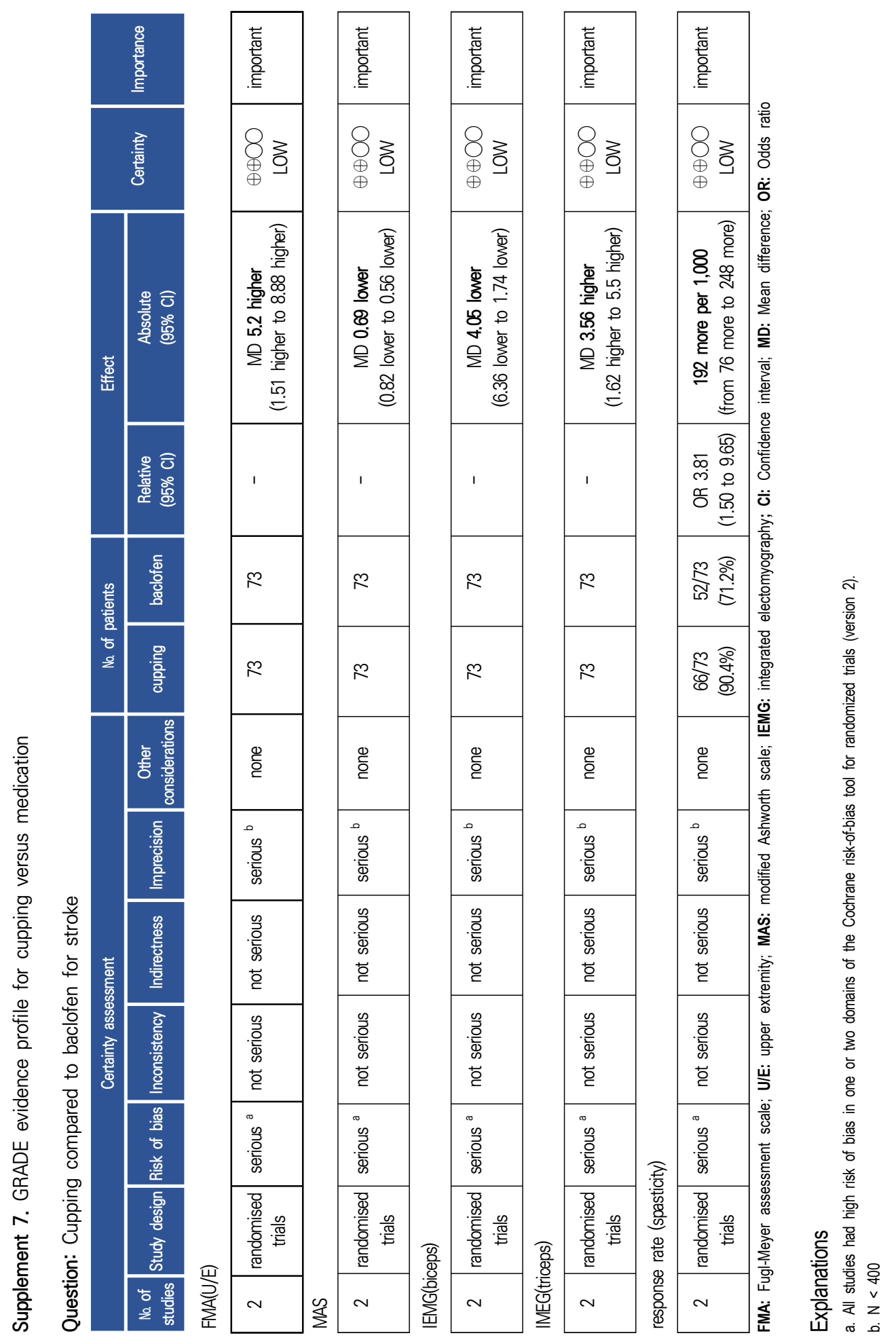




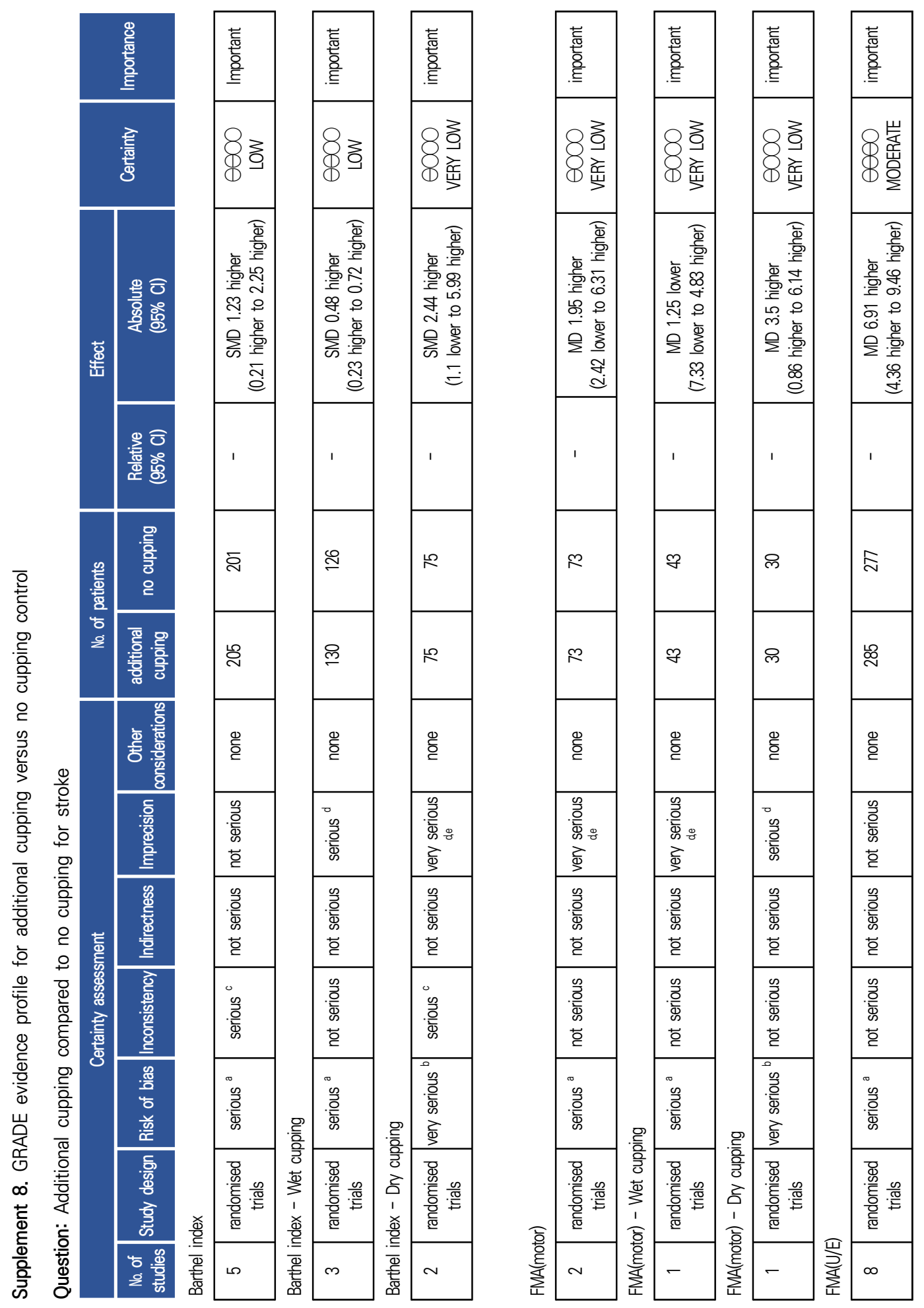




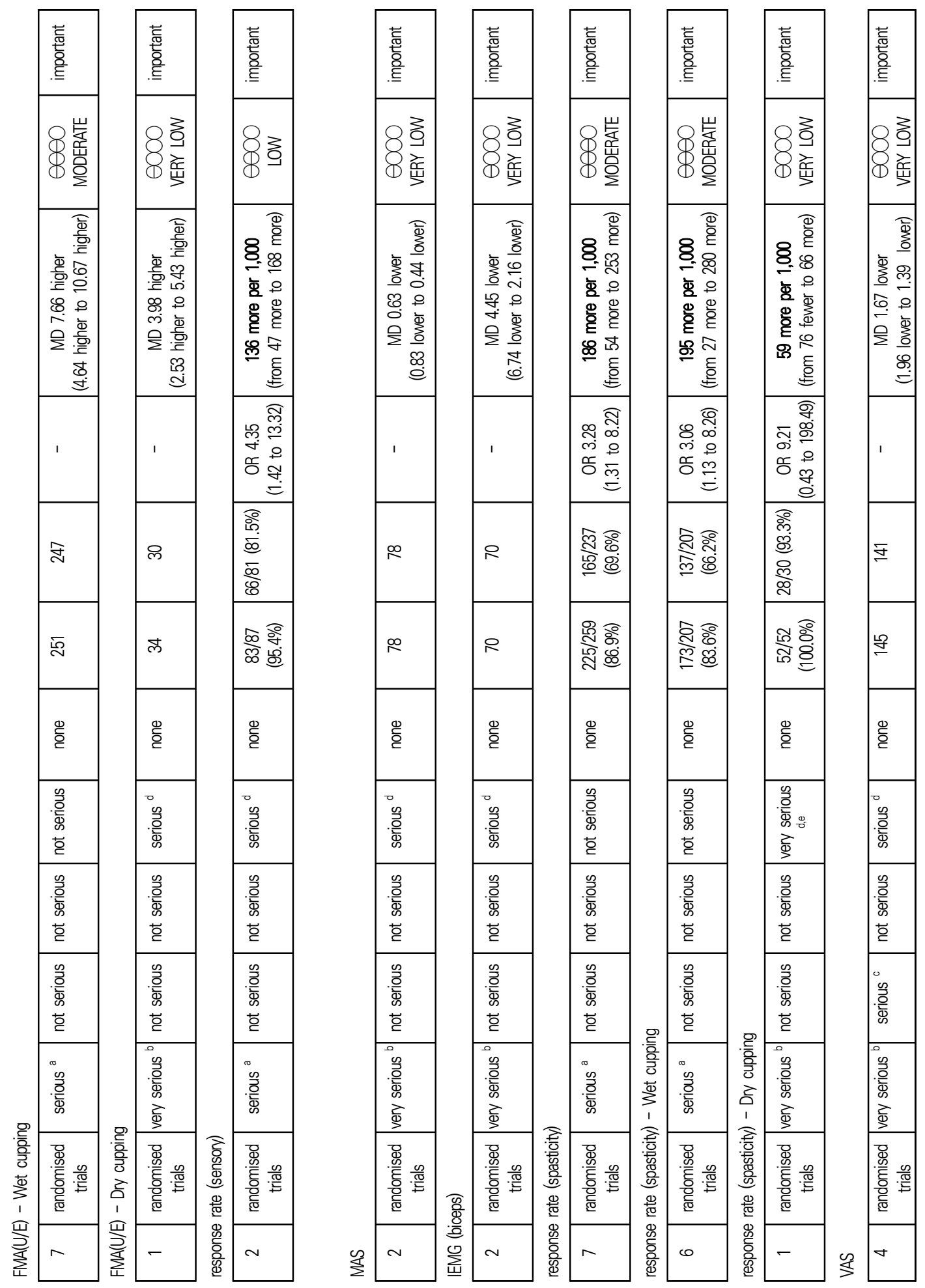




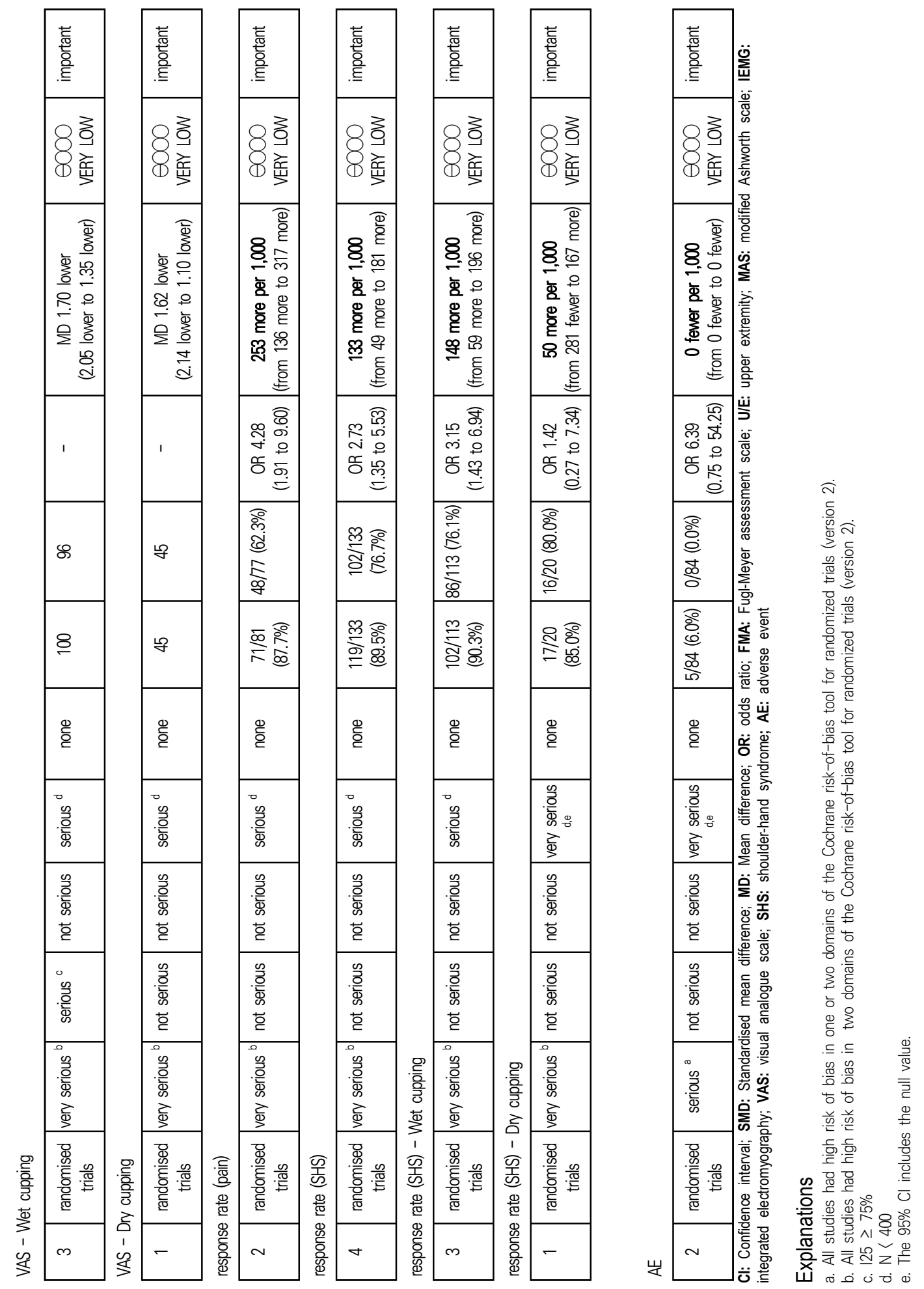

